\title{
Characterization of HNRNPA1 mutations defines diversity in pathogenic mechanisms and clinical presentation
}

\author{
Danique Beijer, ${ }^{1,2}$ Hong Joo Kim, ${ }^{3}$ Lin Guo, ${ }^{4,5}$ Kevin O'Donovan, ${ }^{3}$ Inès Mademan, ${ }^{1,2}$ Tine Deconinck, ${ }^{6}$ \\ Kristof Van Schil, ${ }^{6}$ Charlotte M. Fare, ${ }^{4}$ Lauren E. Drake, ${ }^{4}$ Alice F. Ford, ${ }^{4}$ Andrzej Kochański, \\ Dagmara Kabzińska, ${ }^{7}$ Nicolas Dubuisson, ${ }^{8}$ Peter Van den Bergh, ${ }^{8}$ Nicol C. Voermans, ${ }^{9}$ \\ Richard J.L.F. Lemmers, ${ }^{10}$ Silvère M. van der Maarel, ${ }^{10}$ Devon Bonner, ${ }^{11}$ Jacinda B. Sampson, ${ }^{11}$ \\ Matthew T. Wheeler, ${ }^{11}$ Anahit Mehrabyan, ${ }^{12}$ Steven Palmer, ${ }^{12}$ Peter De Jonghe, ${ }^{1,2,13}$ James Shorter, ${ }^{4}$ \\ J. Paul Taylor, ${ }^{3,14}$ and Jonathan Baets ${ }^{1,2,13}$ \\ ${ }^{1}$ Translational Neurosciences, Faculty of Medicine and Health Sciences, and 'Laboratory for Neuromuscular Pathology, \\ Institute Born-Bunge, University of Antwerp, Wilrijk, Belgium. ${ }^{3}$ Department of Cell and Molecular Biology, St. Jude \\ Children's Research Hospital, Memphis, Tennessee, USA. ${ }^{4}$ Department of Biochemistry and Biophysics, Perelman School \\ of Medicine, University of Pennsylvania, Philadelphia, Pennsylvania, USA. ${ }^{5}$ Department of Biochemistry and Molecular \\ Biology, Sidney Kimmel Medical College, Thomas Jefferson University, Philadelphia, Pennsylvania, USA. ${ }^{6}$ Medical Cenetics, \\ University of Antwerp and Antwerp University Hospital, Edegem, Belgium. ${ }^{7}$ Neuromuscular Unit, Mossakowski Medical \\ Research Centre, Polish Academy of Sciences, Warsaw, Poland. ${ }^{8}$ Neuromuscular Reference Centre, University Hospitals \\ St-Luc, University of Louvain, Brussels, Belgium. ${ }^{9}$ Department of Neurology, Donders Institute for Brain, Cognition and \\ Behaviour, Radboud University Medical Center, Nijmegen, Netherlands. ${ }^{10}$ Human Genetics Department, Leiden University \\ Medical Center, Netherlands. "'Stanford Center for Undiagnosed Diseases, Stanford University, Stanford, California, USA. \\ ${ }^{12}$ Department of Neurology, School of Medicine, University of North Carolina at Chapel Hill, Chapel Hill, North Carolina, \\ USA. ${ }^{13}$ Neuromuscular Reference Centre, Department of Neurology, Antwerp University Hospital, Wilrijk, Belgium. \\ ${ }^{14}$ Howard Hughes Medical Institute, Chevy Chase, Maryland, USA.
}

Authorship note: D Beijer, HJK, LG, $\mathrm{JS}, \mathrm{JPT}$, and JB contributed equally to this work.

Conflict of interest: $J S$ is a consultant for Dewpoint Therapeutics, Maze Therapeutics, Vivid Sciences, Korro Bio, and ADRx.

Copyright: () 2021, Beijer et al. This is an open access article published under the terms of the Creative Commons Attribution 4.0 International License.

Submitted: February 4, 2021

Accepted: June 3, 2021

Published: July 22, 2021

Reference information: JCI Insight. 2021;6(14):e148363. https://doi.org/10.1172/jci. insight.148363.
Mutations in HNRNPA1 encoding heterogeneous nuclear ribonucleoprotein (hnRNP) A1 are a rare cause of amyotrophic lateral sclerosis (ALS) and multisystem proteinopathy (MSP). hnRNPA1 is part of the group of RNA-binding proteins (RBPs) that assemble with RNA to form RNPs. hnRNPs are concentrated in the nucleus and function in pre-mRNA splicing, mRNA stability, and the regulation of transcription and translation. During stress, hnRNPs, mRNA, and other RBPs condense in the cytoplasm to form stress granules (SGs). SGs are implicated in the pathogenesis of (neuro-)degenerative diseases, including ALS and inclusion body myopathy (IBM). Mutations in RBPs that affect SC biology, including FUS, TDP-43, hnRNPA1, hnRNPA2B1, and TIA1, underlie ALS, IBM, and other neurodegenerative diseases. Here, we characterize 4 potentially novel HNRNPA1 mutations (yielding 3 protein variants: *321Eext ${ }^{*} 6,{ }^{*} 321$ Q ext ${ }^{*} 6$, and $\mathrm{C} 304 \mathrm{Nfs}^{*} 3$ ) and 2 known HNRNPA1 mutations (P288A and D262V), previously connected to ALS and MSP, in a broad spectrum of patients with hereditary motor neuropathy, ALS, and myopathy. We establish that the mutations can have different effects on hnRNPA1 fibrillization, liquid-liquid phase separation, and SC dynamics. P288A accelerated fibrillization and decelerated SC disassembly, whereas ${ }^{*} 321$ Eext $^{*} 6$ had no effect on fibrillization but decelerated SC disassembly. By contrast, C304Nfs*3 decelerated fibrillization and impaired liquid phase separation. Our findings suggest different underlying pathomechanisms for HNRNPA1 mutations with a possible link to clinical phenotypes.

\section{Introduction}

Heterogeneous nuclear ribonucleoprotein (hnRNP) A1 is a member of a large class of RNA-binding proteins (RBPs) that assemble with RNA to form ribonucleoproteins (RNPs). hnRNPs are evolutionarily conserved and are primarily localized to the nucleus, where they function in pre-mRNA splicing, mRNA stability, miRNA maturation, regulation of transcription and translation, and telomere biogenesis (1-7). 
Mutations affecting several RBPs, including FUS, TDP-43, hnRNPA1, hnRNPA2B1, matrin-3, and TIA1, are linked to amyotrophic lateral sclerosis (ALS), frontotemporal dementia (FTD), multisystem proteinopathy (MSP), and hereditary inclusion body myopathy (hIBM) phenotypes $(8,9)$.

Missense mutations in the prion-like domain (PrLD) of hnRNPA1 (p.D262V/N and p.N267S) are linked to ALS (MIM 615426) and MSP3 (MIM 615424) (10, 11). Additional ALS-linked mutations in hnRNPA1 are also in the PrLD (p.G264R) (12) or the nuclear localization sequence (p.P288S/A) (13, 14). The p.D262N mutation in HNRNPA1 was also reported in 2 families with IBM with a pure muscular phenotype, illustrating the heterogeneity of consequences that arise from HNRNPA1 mutations. Several modest-sized genetic screenings failed to identify further HNRNPA1 mutations in 2485 patients with ALS, FTD, MSP, hIBM, or other myopathies, suggesting that disease-linked mutations in HNRNPA1 may be rare (15-19).

Stress granules (SGs) are at the nexus of RBP pathology and neurodegenerative diseases (20-27). SGs are cytoplasmic membrane-less organelles that contain RNA and proteins, including several RBPs connected with neurodegeneration (22). SGs mostly contain mRNA transcripts, with approximately $80 \%$ of the SG consisting of mRNAs from essentially every expressed gene $(28,29)$. SGs also specifically contain small ribosomal subunits, translation initiation factors (eIF3, eIF4E, eIF4G), hnRNPs, and other RBPs, such as TIA1, HuR, and PABP (30). SGs accumulate upon stress via the formation and fusion of numerous small condensates (31). Importantly, SGs dissolve once stress has passed, allowing SG components to return to performing their soluble function $(31,32)$. As a subcellular compartment, SGs are central to the pathogenesis of degenerative diseases, as disease-causing mutations in RBPs are associated with accumulation of persistent SGs in ALS, FTD, and IBM (10, 21, 23, 26, 33, 34).

Neurodegenerative diseases are consistently characterized by pathological proteinaceous inclusions, a subset of which contain SG markers $(8,9,35,36,37)$. A good example is the deposition of TDP-43, which is a prominent feature of approximately $97 \%$ of ALS cases, as well as nearly all cases of sporadic IBM and hIBM $(10,24,35-37)$. Pathological inclusion formation may be due to the failure of SGs to disassemble (26). It is critical to distinguish whether inclusions observed in postmortem tissues represent persistent SGs or partially disassembled SGs or SG proteins are recruited to preformed inclusions, or some combination of these possibilities (20). Indeed, the precise mechanisms by which specific RBPs become depleted from the nucleus and accumulate in cytoplasmic aggregates remain unclear.

Recent work on the broader group of ventral horn, peripheral nerve, and muscle diseases — including inherited peripheral neuropathy (IPN), hereditary motor neuropathy (HMN), and ALS — has demonstrated a significant genetic overlap, including both pleiotropy and genetic heterogeneity. This overlap is illustrated by findings where causative genes do not exclusively give rise to the phenotype for which they were initially described, as for mutations in SETX, DCTN1, and SIGMAR1, which underlie both ALS and HMN (38-40).

Here, we present patients with 4 potentially novel HNRNPA1 mutations (yielding 3 protein variants: *321Eext*6, *321Qext*6, and G304Nfs*3) and 2 known HNRNPA1 mutations (P288A and D262V). These mutations expand the genetic and clinical spectrum of hnRNPA1-associated neurodegenerative diseases by demonstrating their involvement in complex IPN, atypical ALS, and myopathy. We establish that the mutations can have different effects on hnRNPA1 fibrillization, liquid-liquid phase separation (LLPS), and SG dynamics, indicating the possibility of multiple underlying pathomechanisms for HNRNPA1 mutations with a possible link to the clinical phenotypes.

\section{Results}

Identification of HNRNPA1 mutations in 6 separate families

Mutations in the HNRNPA1 gene can result in a range of neurodegenerative disorders $(8,10,13-15,17$, 41-43). For example, in a patient from family A with motor neuropathy, a heterozygous de novo splice site variation (c.908-2A $>\mathrm{G}$ ) upstream of the last exon of HNRNPA1 was identified (Figure 1). cDNA analysis in this patient by PCR and Sanger sequencing revealed skipping of the last exon, producing a transcript that was not degraded by nonsense-mediated decay (NMD), resulting in a truncated protein (p.G304Nfs*3) (Figures 1 and 2 and Supplemental Figure 1; supplemental material available online with this article; https://doi.org/10.1172/jci.insight.148363DS1). To screen for additional hnRNPA1 mutations in patients, we surveyed a large cohort of 547 patients with distal HMN (dHMN), axonal Charcot-Marie-Tooth disease (CMT2), intermediate CMT (CMTi), spinal muscular atrophy (SMA), ALS, hereditary spastic paraplegia (HSP), and myopathy. Thus, we identified a missense variation (c.862C > G; p.P288A) in the index patient 


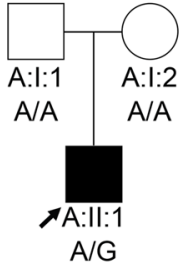

c. $908-2 A>G$ (p.G304Nfs*3)

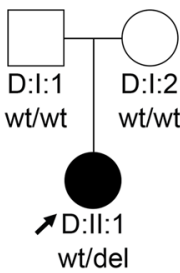

500bp deletion (p.G304Nfs*3)
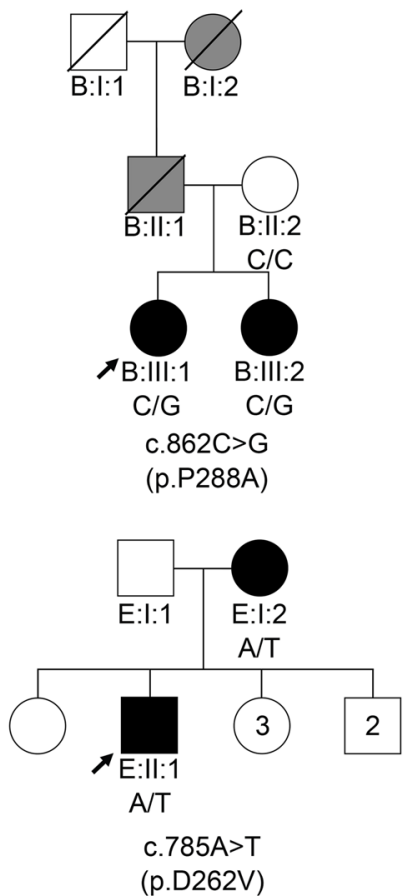
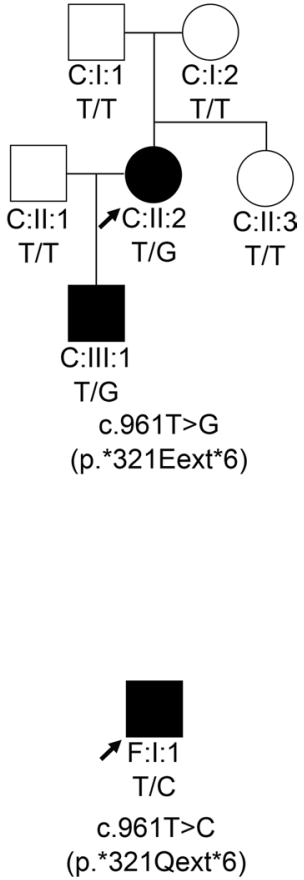

Figure 1. Heterozygous HNRNPA1 mutations in 6 families. Pedigrees of families A-F with their respective mutations and the segregation of each by genotype, showing affected (black), unaffected (white), and hearsay-affected (gray) individuals. Arrows indicate probands.

of family B that has been linked to ALS (Figure 1) (14). Whole-exome sequencing (WES) for this family did not reveal segregating mutations in other genes known for motor neuron diseases or related disorders. Therefore, the P288A mutation is the most likely cause of disease in family B.

Four additional HNRNPA1 mutations were identified in families C-F (Figure 1). Whole-genome sequencing (WGS) in family $\mathrm{C}$ revealed a single-nucleotide variant in the HNRNPA1 stop-codon (c.961T $>\mathrm{G}$; p. ${ }^{*} 321 \mathrm{E}$ ext* ${ }^{*}$ ) resulting in a stop-loss mutation without undergoing NMD. This mutation added 6 extra amino acids to the protein before ending at an alternative stop-codon (Figure 2). Both this stop-loss mutation and the splice variant from family A terminate on the same TAG sequence present in the WT 3'-UTR (Figure 2). In family D, a 500 bp deletion in HNRNPA1 was identified through trio WGS (Supplemental Figure 1D). This variant results in the same mRNA alteration as the variant in family A, yielding the same mutant protein (p.G304Nfs*3). For family E, WES in individual E:II:2 identified a known HNRNPA1 (c.785A>T; p.D262V) missense mutation, which was previously reported in a family with autosomal dominant myopathy and Paget's disease of bone (PDB) $(10,42)$. We also identified a nearly identical mutation to *321Eext*6 linked to a similar phenotype in the isolated index of family F (p.*321Qext*6) (Figures 1 and 2 and Supplemental Table 2).

\section{HNRNPA1 mutations are linked to a variety of phenotypes}

Family $A-\left(p . G 304 N f f^{*} 3\right)$. At 15 years of age, the isolated Polish patient (A:II:1) presented the first symptoms with wasting of hand intrinsics. At 16, sural nerve biopsy showed very mild axonal neuropathy with secondary segmental demyelination. Electromyography (EMG) in median, ulnar, and peroneal nerves showed decreased amplitudes of compound muscle action potentials (CMAPs) with nerve conduction velocities in the normal range, while no abnormalities were observed in sensory fibers. At 24 , he displayed right-sided thoracic scoliosis, mild pes cavus, wasting of the forearms and calves (lower third), and brisk deep tendon reflexes in the upper and lower limbs with intact sensory modalities. By age 40 , he had severe wasting of the distal muscles of the upper and lower limbs. Despite a very clumsy gait with foot drop, he is still ambulant. Overall, his phenotype corresponds to an axonal motor-predominant neuropathy (dHMN).

Family B - (p.P288A). Patient B:II:1 is a 44-year-old woman of Moroccan origin who developed painless proximal right arm weakness and wasting without sensory disturbances at age 22. Her father and paternal grandmother presented the same phenotype. Both died from respiratory failure around the age of 40 . She was diagnosed with a very slowly progressive atypical ALS 4 years after symptom onset, based on the clinical 


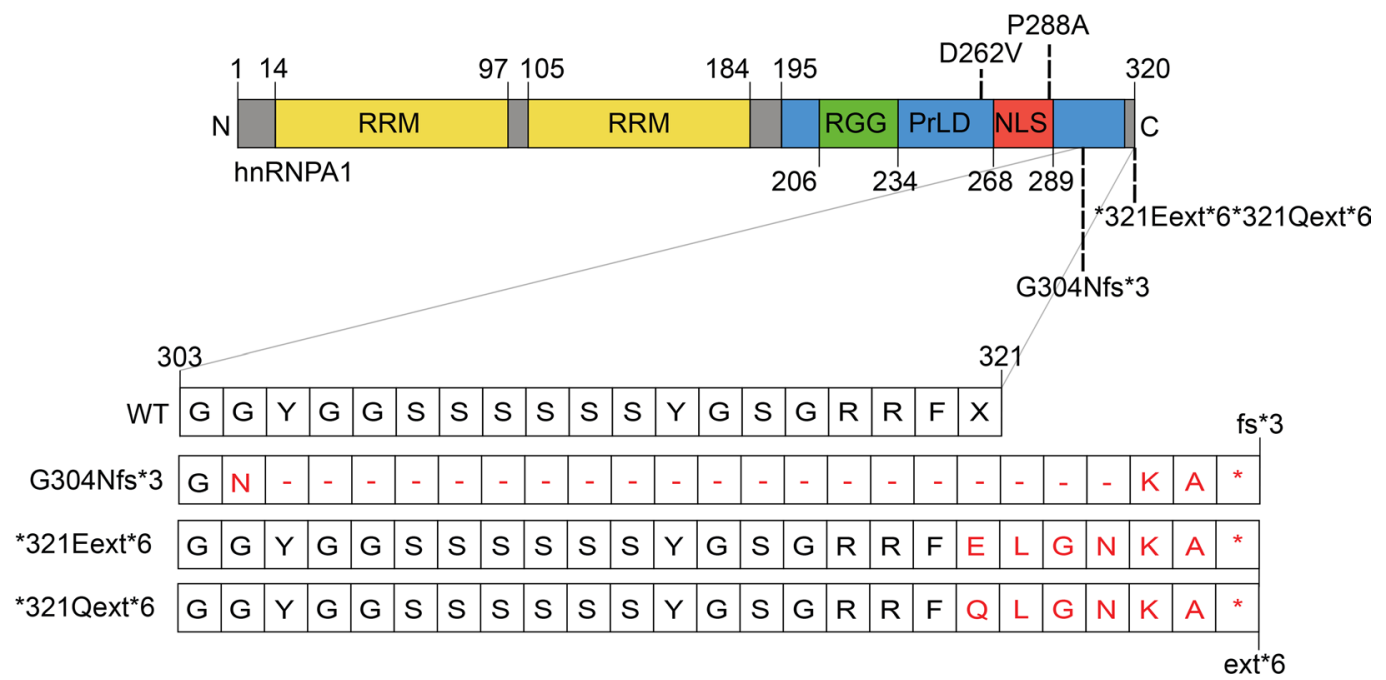

Figure 2. Schematic overview of hnRNPA1 protein and mutations. hnRNPA1 contains $2 \mathrm{~N}$-terminal RNA-recognition motifs (RRMs) and a C-terminal prion-like domain (PrLD), containing an arginine-glycine-glycine (RGG) motif and a proline-tyrosine nuclear localization signal (PY-NLS) that enables nuclear import. Mutations studied in this paper are shown. The effects of the frameshift and extension $\mathrm{G} 304 \mathrm{Nfs}^{*} 3$, ${ }^{*} 321 \mathrm{Eext}{ }^{*} 6$, and ${ }^{*} 321$ Q ext ${ }^{*} 6$ mutations at the protein level are shown in the zoom-in of amino acids 303-321.

features with supportive neurophysiological findings: upper and lower motor neuron symptoms observed at the clinical examination and a chronic neurogenic pattern limited to the upper limb and tongue muscles recorded via EMG. Over time, she developed progressive weakness and wasting of the left arm, respiratory insufficiency, and lower limb spasticity. Five years after symptom onset, she developed bulbar symptoms with dysarthria, rhinolalia, chewing difficulties, and eventually dysphagia for both liquids and solids. She required percutaneous endoscopic gastrostomy 3 years later. After 22 years of disease progression, she is still walking without aid. Her 3 children are unaffected at the ages of 16, 21, and 25. At age 43, her older sister (patient B:II:2) presented with progressive bulbar symptoms, including dysarthria, tongue atrophy, and swallowing problems. She also presented upper motor neuron signs and right hand weakness with amyotrophy. Combined, the phenotype in this family is that of an atypical and slowly progressive ALS.

Family $C-\left(p *{ }^{*} 321 E\right.$ ext $\left.{ }^{*} 6\right)$. Patient C:II:2 is of Dutch origin and showed her first symptoms at 9 years of age. She presented with hand function difficulties, mild facial weakness, and general fatigue by the age of 12. Muscle weakness and atrophy were gradually progressive. She has been using a wheelchair since age 34 . At age 45, muscle strength in upper and lower limbs had severely decreased, and reflexes were absent in all limbs. She also had a very severe facial weakness and subsequent dysarthria. An quadriceps muscle biopsy was performed, showing nonspecific myopathic changes with some angular fibers but no type grouping. Nerve conduction studies (NCSs) were normal apart from small CMAPs due to severe distal atrophy. Needle EMG was compatible with a myogenic process. The patient developed a mild dissociated vital hyposensitivity distally in the legs, but no abnormalities were noted on spinal MRI. The clinical diagnosis of facio-scapulo-humeral muscular dystrophy could not be confirmed genetically.

The patient's son (C:III:1) presented his first symptoms with a right-sided foot drop at the age of 8 . At 13 , he is ambulant and can still walk on his toes. However, heel walking is impossible. He shows slight wasting of the left thenar and reduced muscle strength in the lower limbs. His reflexes are normal in all limbs. NCSs were normal and needle EMG revealed myopathic features. A needle biopsy of the quadriceps muscle was performed at age 8 , which showed mild and nonpathognomonic myopathic features. The phenotype in this family is compatible with a myopathy with distal and facial onset and severe proximal and bulbar weakness upon progression. The distal weakness, atrophy, and subsequent small CMAPs suggested an overlap with the dHMN disease spectrum, but myopathic features initially dominated the phenotype.

Family D - (p.G304Nfs*3). Individual D:II:1 is a 35-year-old woman of Asian Indian origin who developed right hand weakness, pain when writing, and atrophy at age 22 . At age 26, she was thought to have multifocal motor neuropathy based on her EMG but was treated with a trial of intravenous immunoglobin without response. Her muscle biopsy at age 26 revealed vacuolar myopathy, notable for its myofibrillar 
Table 1. Summary of the molecular features of hnRNPA1 mutants

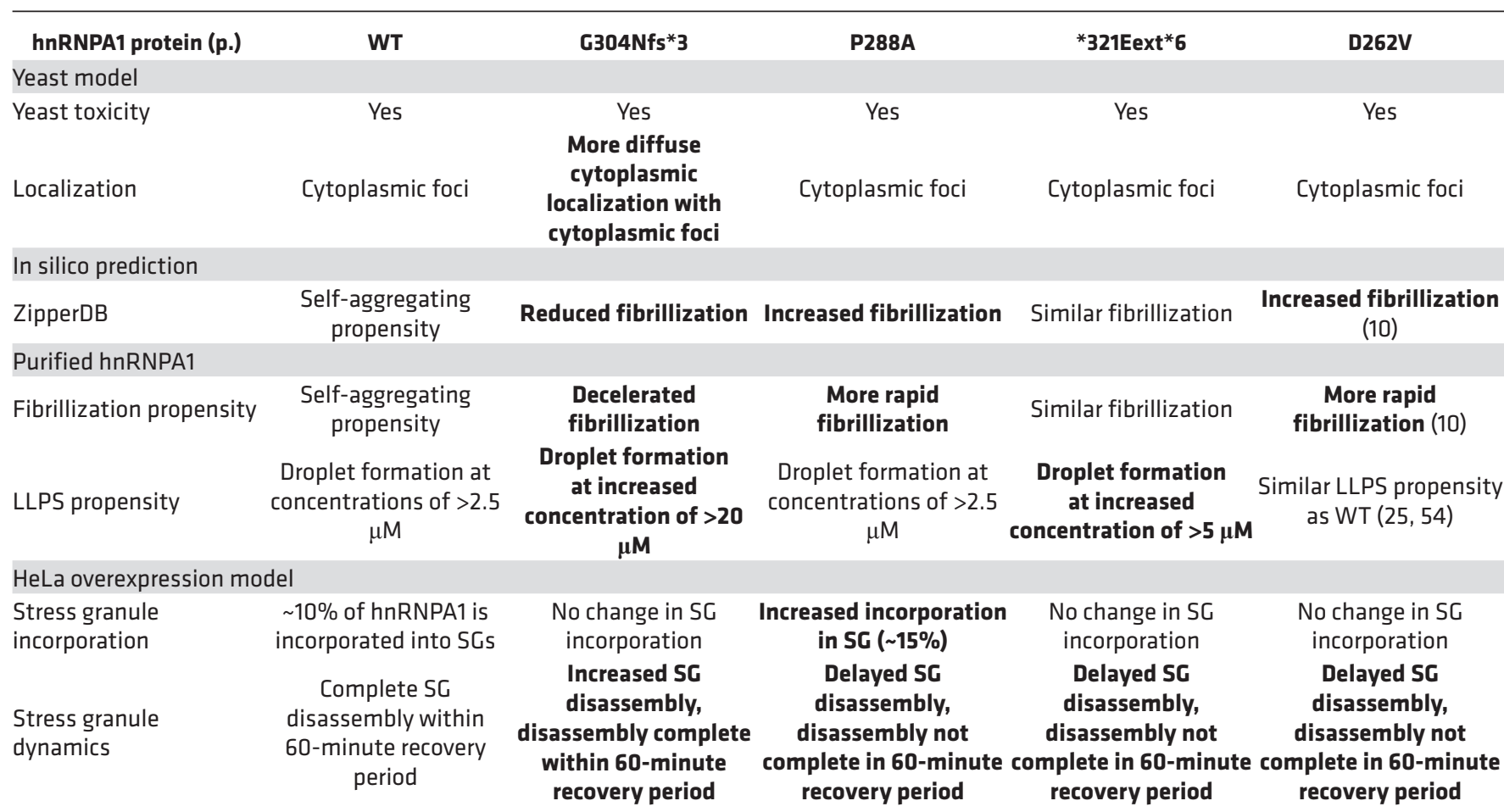

Changes compared with WT are indicated in bold; literature references are presented for experiments not performed in this study.

disorganization, moth-eaten fibers, rimmed vacuoles, and absence of inflammation. Repeat EMG at age 29 showed irritative distal myopathy. Her symptoms have been gradually progressive: at age 27, she noted right ankle weakness, which is now bilateral. Her exam is notable for mild right facial weakness, and her distal weakness is markedly asymmetric. Reflexes were initially preserved in the lower extremities but have diminished over time; there were no other upper motor neuron signs. She walks unassisted, has scapular winging, and has mild swallowing difficulties with normal speech. She has a restrictive lung function with predicted forced vital capacity of $76 \%$ and sleep-disordered breathing. Her creatine kinase has been 2 -fold elevated. Cardiac MRI, electrocardiogram, and 14-day cardiac rhythm monitor were normal.

Family E - (p.D262V). Individual E:II:2 is an American male who developed asymmetric weakness of the right foot at around 36 years of age, worsening over time to involve bilateral proximal leg weakness. He initially noticed difficulties with snow skiing and later had frequent tripping and falls. Over the course of 10 years, there was slow progression of bilateral leg weakness that now requires use of a cane for ambulation. Currently, at age 48, the patient uses his arms to stand from sitting position and requires handrails when going up the stairs. He reports no weakness of facial, neck, arm, or trunk muscles; numbness or tingling; dysphagia; difficulty with handwriting, handling utensils, or opening doors; shortness of breath; bowel/ bladder dysfunction; diplopia; or muscle pain. NCS abnormalities were limited to low amplitude of the right fibular CMAPs, whereas needle EMG revealed chronic myopathic changes proximally and distally in the leg. A muscle biopsy at age 45 showed chronic myopathy with rimmed vacuoles.

The patient's mother (E:I:2) was diagnosed with PDB at the age of 48 . Within 3 years she developed left leg weakness that gradually progressed to involve both legs. She is currently using a wheelchair. She has normal arm strength and normal cranial nerve function, and a muscle biopsy was consistent with myopathy with inclusion bodies. The index patient has 6 unaffected siblings (34-50 years old). The overall phenotype in this family is consistent with inclusion body myositis.

Family F- $\left(p * 321\right.$ Qext $\left.{ }^{*} 6\right)$. Patient F:I:1 is of Belgian origin and presented first at the age of 12 years with muscle atrophy in the hands, with subsequent progressive wasting of the lower limbs starting distally before also involving proximal muscles. At his last examination at the age of 64 , this patient used a wheelchair with profound generalized weakness resulting in de facto quadriplegia. Reflexes were lost in all 
extremities. In addition to contractures in the lower limbs and bilateral pes equinovarus, the patient displayed marked facial weakness. Respiration was severely limited, requiring cough assist and noninvasive home ventilation (bilevel positive airway pressure). The patient also made use of a percutaneous endoscopic gastrostomy. A biopsy of the tibialis anterior muscle at the age of 13 years showed myogenic alterations consisting of marked diameter variation, selective type 1 fiber atrophy, increased centralized nuclei, and the presence of rimmed vacuoles. A biopsy of the fibularis superficialis nerve was strictly normal. NCSs and needle EMG did not show neurogenic changes and were consistent with a distally predominant myopathy. The patient died at age 64. The family history for this patient is negative for neuromuscular disorders, making this an isolated case. The clinical presentation fits with a progressive myopathy with onset in the distal limbs. A clinical diagnosis of distal myopathy of Welander type or Nonaka type was suggested. No mutation in UDP- $N$-acetylglucosamine-2-epimerase/ $N$-acetylmannosamine kinase (GNE) was found.

\section{Comparable in vitro transcription and translation of hnRNPA1 mutants}

To verify that the alternatively terminating variants were not subject to NMD, mRNA sequences for the G304Nfs*3 (family A) and *321Eext*6 (family C) variants were assessed by PCR amplification from exon 8 into the 3 '-UTR on cDNA from patient lymphoblasts. Gel electrophoresis and di-deoxy sequencing confirmed exon 11 skipping for the G304Nfs*3 splice variant (Supplemental Figure 1A). mRNA for the G304Nfs*3 and *321Eext*6 variants was present, indicating the absence of NMD and suggesting that these genes are transcribed and likely translated in patients (Supplemental Figure 1, B and C). RNA sequencing confirmed altered splicing in family D, resulting in the same protein as family A (Supplemental Figure 1D). Next, hnRNPA1 plasmids were constructed for N-terminally V5 tagged WT hnRNPA1 cDNA, as well as P288A, G304Nfs*3, and *321Eext*6 mutations, and each protein was transiently expressed in Henrietta Lacks (HeLa) cells. Immunoblotting confirmed translation of WT hnRNPA1 and the mutant proteins. As expected, the G304Nfs*3 mutation produced a shorter protein, whereas the ${ }^{*} 321 \mathrm{Eext} * 6$ mutation yielded a longer protein (Supplemental Figure 1E). The P288A mutation translated into a protein of similar size to WT hnRNPA1 (Supplemental Figure 1E). Thus, these patient-derived mutant mRNA transcripts can be translated into mutant hnRNPA1 protein in cells.

\section{WT and mutant hnRNPA1 confer toxicity in yeast}

Next, we assessed the hnRNPA1 variants in a yeast model of hnRNPA1 aggregation and toxicity (10). The hnRNPA1 PY-NLS is not decoded by the yeast nuclear import machinery, and hnRNPA1 localizes to the cytoplasm in yeast (44-46). hnRNPA1 and hnRNPA1 ${ }^{\mathrm{D} 262 \mathrm{~V}}$ form cytoplasmic aggregates in yeast and are toxic, which phenocopies pathological events in MSP $(10,47)$. Here, it is important to note that in MSP cases caused by mutations in $V C P$ or hnRNPA2, it is the WT version of hnRNPA1 that undergoes cytoplasmic aggregation (10). Thus, expression of WT hnRNPA1 in yeast recapitulates the cytoplasmic mislocalization and aggregation of WT hnRNPA1 that occurs in MSP caused by mutations in hnRNPA2 or VCP (10).

We transformed WT or mutant hnRNPA1 on a galactose-induced expression vector and serially diluted yeast onto glucose (no expression of hnRNPA1), sucrose/galactose (1:1; moderate expression of hnRNPA1), or galactose-containing agar plates (high expression of hnRNPA1). Expression of WT or mutant hnRNPA1 on sucrose/galactose (1:1) and galactose media was toxic (Figure 3A). Immunoblot confirmed that hnRNPA1 variants were robustly expressed in yeast on galactose media (Figure 3, B and C). Thus, in yeast, the hnRNPA1 variants displayed similar toxicity to WT hnRNPA1, suggesting that the cytoplasmic localization of hnRNPA1 is pathogenic for these variants in yeast (Figure 3A).

Next, we assessed the aggregation of mutant hnRNPA1. hnRNPA1, hnRNPA1 ${ }^{\text {D262V }}$, hnRNPA1 ${ }^{\text {P288A }}$, and hnRNPA1 $1^{* 321 \text { Ext*6 }^{*}}$ formed cytoplasmic foci (Figure 3D). By contrast, hnRNPA1 ${ }^{\mathrm{G} 304 \mathrm{Nfs}{ }^{* 3}}$ displayed more diffuse localization, although cytoplasmic foci were still evident (Figure 3D). G304Nfs*3 was expressed to similar levels as the other hnRNPA1 proteins (Figure 3, B and C). Thus, hnRNPA1 $1^{\mathrm{G} 304 \mathrm{Nfs}^{* 3}}$ may be less aggregation prone than hnRNPA1, hnRNPA1 ${ }^{\text {D262V }}$, hnRNPA1 ${ }^{\text {P288A }}$, and hnRNPA1 ${ }^{\star_{321 E x t^{*} 6}}$ while conferring similar toxicity (Figure $3 \mathrm{~A}$ ).

\section{Differential fibrillization and LLPS propensity of hnRNPA1 variants}

Our yeast work suggested that hnRNPA1 variants may differ in their propensity to self-assemble. To investigate how the mutations affect the fibrillization propensity of hnRNPA1, we first examined WT hnRNPA1 and the disease-associated mutants with ZipperDB (48). ZipperDB is a structure-based threading algorithm, which scores 6 amino acid segments for their propensity to form 2 self-complementary $\beta$-strands 


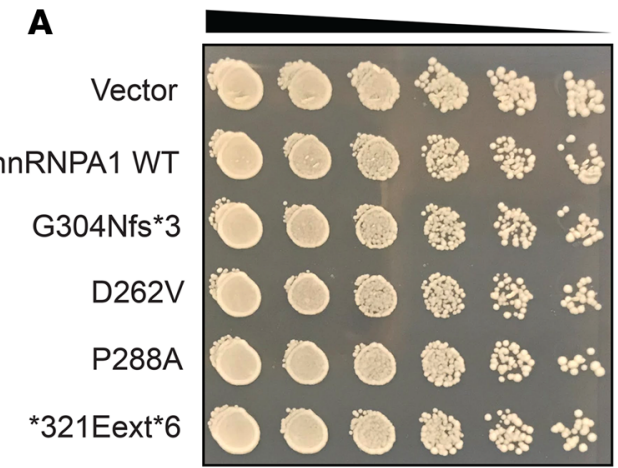

Glucose

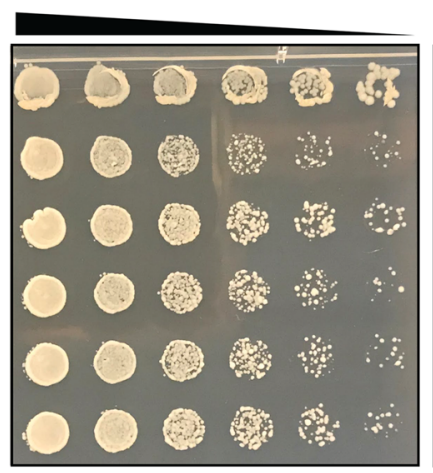

Galactose:Sucrose (1:1)

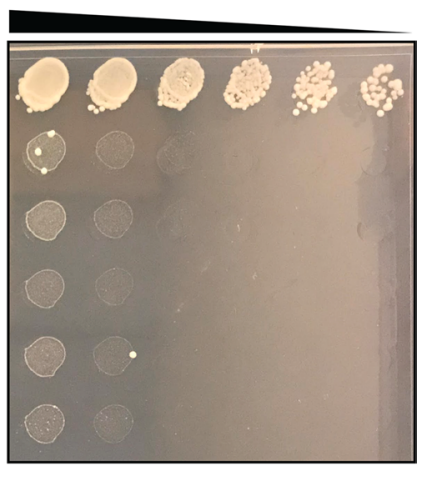

Galactose
B

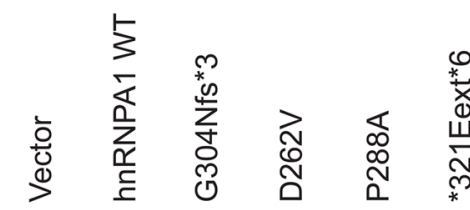

75

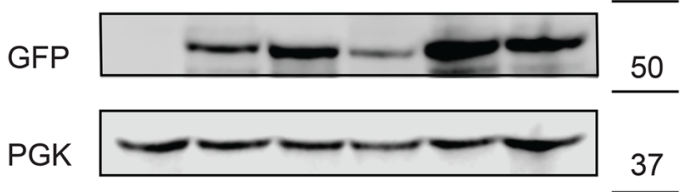

C

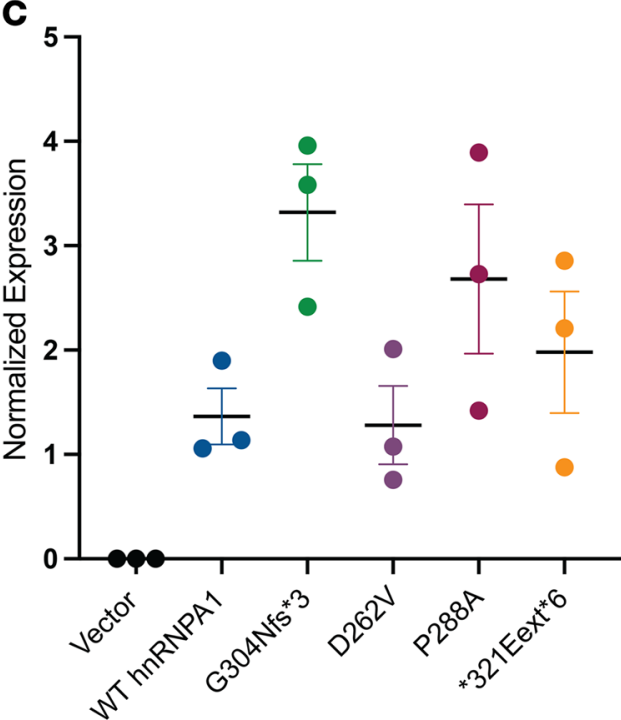

D

hnRNPA1 WT
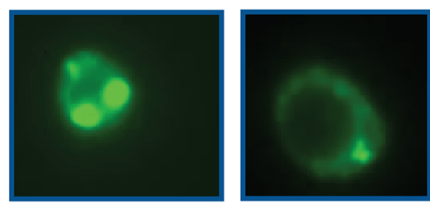

G304Nfs*3
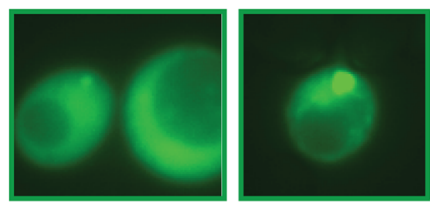

D262V
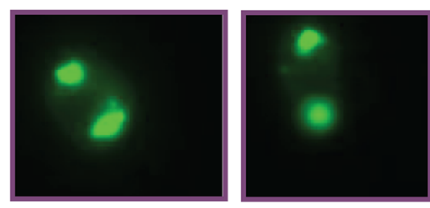

P288A
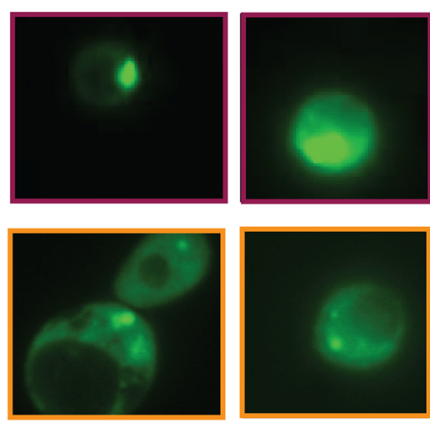

$5 \mu \mathrm{m}$

Figure 3. hnRNPA1 variants are toxic and aggregate in yeast. (A) GFP-tagged hnRNPA1 variants are toxic when expressed in yeast. Yeast spotting assays compare the toxicity of hnRNPA1 variants by plating yeast at serially diluted concentrations. hnRNPA1 variants were expressed from a galactose-inducible promoter for 72 hours. Expression of hnRNPA1 is repressed when cells are grown on glucose (left panel), then moderately induced on sucrose/galactose (middle panel), and strongly induced on galactose (right panel). (B) Western blot confirms the expression of GFP-tagged hnRNPA1 variants in yeast after 8 hours in galactose. Phosphoglycerate kinase (PGK) is used as loading control. (C) GFP signal was quantified from 3 separate blots and normalized to the PGK signal of the respective sample. The variability in hnRNPA1 expression is not significant as measured by 1-way ANOVA using Dunnett's multiple comparisons test. (D) Yeast cells expressing GFP-tagged WT and mutant hnRNPA1. hnRNPA1, hnRNPA1 ${ }^{\text {D262V }}$, hnRNPA1 ${ }^{\text {P288A }}$, and hnRNPA1 ${ }^{* 321 E e x t^{*} 6}$ formed cytoplasmic foci. hnRNPA1 ${ }^{\mathrm{CBOANfs}^{*} 3}$ displayed more diffuse localization in yeast, although cytoplasmic foci could be found.

termed "steric zippers" that form the spine of amyloid fibrils. Hexapeptides with Rosetta energy of lower than $-23 \mathrm{kcal} / \mathrm{mol}$ are predicted to form steric zippers, with lower energy predicting higher amyloidogenicity. ZipperDB predicted that the P288A mutation introduces a new steric zipper (285-SSGAYG-290) that could increase the fibrillization propensity (Figure 4A). By contrast, G304Nfs*3 deletes a large stretch of 


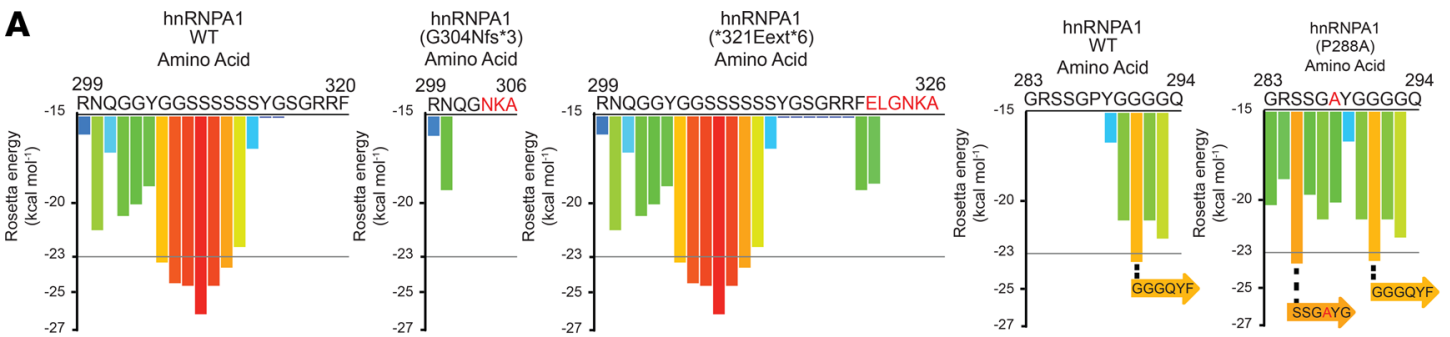

B

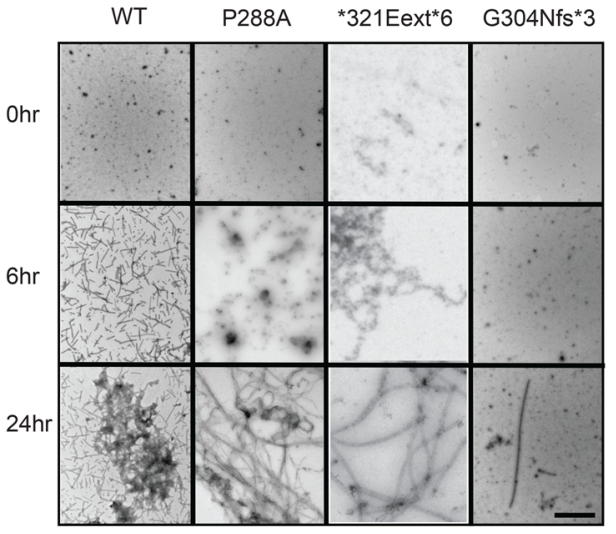

C

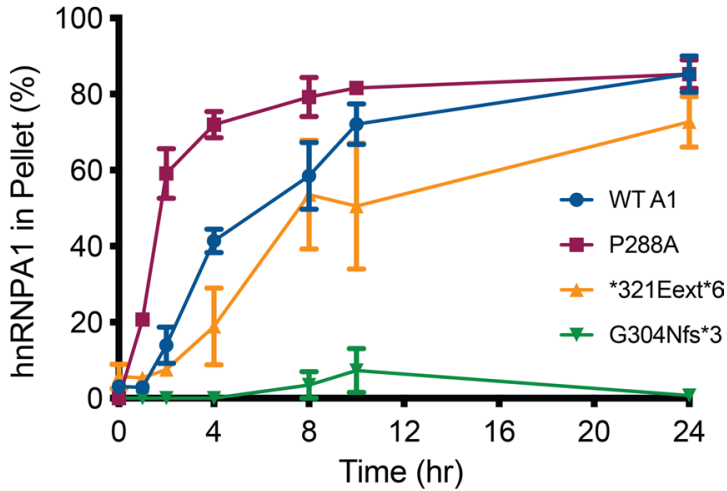

D

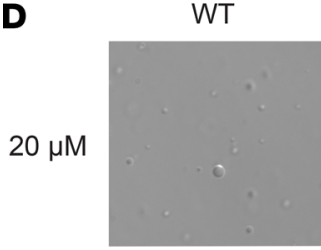

$15 \mu \mathrm{M}$
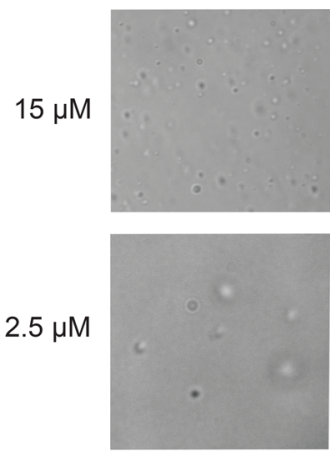

P288A
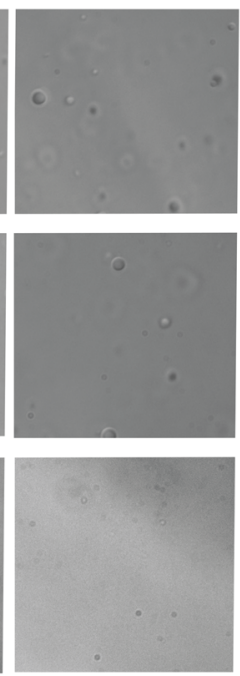

*321Eext*6
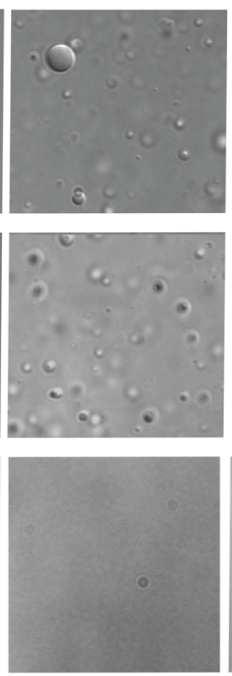

G304Nfs*3

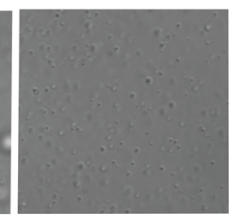

E

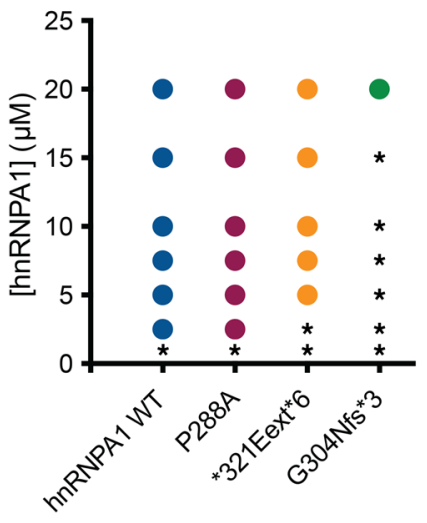

Figure 4. hnRNPA1 variants can exhibit altered propensity for fibrillization and LLPS. (A) ZipperDB calculates the propensity of hexapeptide fragments to form steric zippers (48). Steric zippers, which are self-complementary $\beta$-sheets that form the backbone of amyloid fibrils, are predicted to form when the Rosetta energy of a hexapeptide is below the empirically determined "high fibrillization propensity" threshold of -23 kcal/mol (48). P288A introduces a potent steric zipper (285-SSGAYG-290) that could increase the fibrillization propensity. G304Nfs*3 deletes several potent steric zippers, which could reduce fibrillization propensity. (B and C) GST-TEV-hnRNPA1 and disease variants ( $5 \mu \mathrm{M})$ were incubated with TEV protease in A1 assembly buffer to initiate fibrillization. Reactions were agitated at $1200 \mathrm{rpm}$ or $0-24$ hours at $25^{\circ} \mathrm{C}$. Fibrillization was monitored by electron microscopy (B). Scale bar, 0.5 $\mu \mathrm{m}$. Alternatively, hnRNPA1 fibrillization kinetics were determined by sedimentation analysis (C) where the amount of hnRNPA1 in the pellet fraction was quantified. Values represent average \pm SEM $(n=3-6)$. (D) Representative differential interference contrast (DIC) microscopy images of hnRNPA1 droplets formed by different hnRNPA1 variants. Droplets were formed by combining the indicated hnRNPA1 variant at the indicated concentration in a LLPS buffer and were imaged immediately after all components had been added. Scale bar: $25 \mu \mathrm{m}$. (E) Phase diagram of hnRNPA1 variants showing the hnRNPA1 concentrations where LLPS occurs. hnRNPA1 and hnRNPA1 ${ }^{\text {P288A }}$ form droplets at concentrations of $2.5 \mu$ M or higher. hnRNPA1 ${ }^{* 321 \text { Ext*6 }}$ forms droplets at concentrations of $5 \mu \mathrm{M}$ or higher. hnRNPA $1^{\mathrm{C304Nfs} * 3}$ forms droplets at concentrations of $20 \mu \mathrm{M}$ or higher. At each concentration, a colored circle indicates droplet formation, whereas a black asterisk indicates no droplets. 
steric zippers present in the C-terminal portion of the PrLD, which could reduce multivalency and aggregation propensity (Figure 4A). Finally, the *321Eext*6 mutation did not change the steric zipper landscape, with ZipperDB predicting a similar fibrillization propensity to WT hnRNPA1 (Figure 4A).

To assess the fibrillization propensity, we purified GST-TEV-tagged versions of the proteins from $E$. coli (10). Fibrillization of purified GST-TEV-hnRNPA1 was initiated by incubating with TEV protease to remove the GST tag (10). Fibrillization kinetics were then monitored by sedimentation analysis, and the final structures that formed were imaged via electron microscopy (10). hnRNPA1 ${ }^{\mathrm{P} 288 \mathrm{~A}}$ assembled into fibrils more rapidly than WT hnRNPA1 (Figure 4, B and C), consistent with the ZipperDB prediction (Figure 4A). Thus, P288 disfavored $\beta$-sheet interactions between hnRNPA1 proteins that drive fibrillization (Figure 4A) (4951). $\mathrm{P} 288$ is also a critical component of the hnRNPA1 PY-NLS, which is important for nuclear localization $(52,53)$. Therefore, in cells, the P288A mutation contributes to increased cytoplasmic hnRNPA1 mislocalization and aggregation. As predicted by ZipperDB, hnRNPA $1^{{ }^{* 321 E e x *^{*} 6}}$ exhibited similar fibrillization kinetics to WT hnRNPA1 (Figure 4, B and C). Strikingly, hnRNPA1 ${ }^{\mathrm{G} 304 \mathrm{Nfs}{ }^{* 3}}$ displayed decelerated fibrillization, with only a few fibrils apparent after 24 hours (Figure 4, B and C), again in line with ZipperDB predictions (Figure $4 \mathrm{~A}$ ) and reduced formation of cytoplasmic foci in yeast (Figure 3D). Thus, P288A accelerates hnRNPA1 fibrillization, whereas $* 321 \mathrm{Eext} * 6$ has minimal effect, and G304Nfs*3 reduces fibrillization.

hnRNPA1 is a component of SGs, which form via LLPS $(10,25)$. Similar to other PrLD-containing RBPs in SGs, purified hnRNPA1 forms liquid droplets spontaneously upon macromolecular crowding (25). In the presence of a crowding agent, WT hnRNPA1 forms liquid droplets at concentrations of $2.5 \mu \mathrm{M}$ or greater (Figure 4, D and E). hnRNPA1 ${ }^{\text {P288A }}$ showed similar LLPS properties to WT hnRNPA1 (Figure 4, D and E), whereas hnRNPA1 $1^{* 321 \mathrm{Eext}^{*} 6}$ had slightly reduced ability to undergo LLPS, as no droplets formed at $2.5 \mu \mathrm{M}$ (Figure 4, D and E). Thus, the features that drive fibrillization of hnRNPA1 (such as steric zippers) do not necessarily have the same effect on LLPS (54). By contrast, hnRNPA1 ${ }^{\mathrm{G} 304 \mathrm{Nf} * 3}$ exhibited reduced LLPS as liquid droplets were only observed at $20 \mu \mathrm{M}$ (Figure $4, \mathrm{D}$ and E). Moreover, hnRNPA1 ${ }^{\mathrm{G} 304 \mathrm{Nfs}{ }^{\star 3}}$ droplets were smaller than those formed by hnRNPA1, hnRNPA1 ${ }^{\text {P288A }}$, and hnRNPA1*321Eext*6 (Figure 4, D and E). Thus, the G304Nfs*3 mutation perturbs hnRNPA1 LLPS and fibrillization, likely by reducing PrLD multivalency (Figure 4A).

\section{hnRNPA1 mutants modulate SG dynamics}

In human cells, hnRNPA1 localizes to SGs in response to specific stressors $(10,55,56)$. Thus, we expressed WT or mutant GFP-tagged hnRNPA1 in HeLa cells and subjected these cells to environmental stressors. WT hnRNPA1 was predominantly nuclear upon arsenite stress (Figure $5, \mathrm{~A}$ and B). G304Nfs*3 and *321Eext*6 mutations did not affect incorporation of hnRNPA1 into arsenite-induced SGs, whereas hnRNPA1 $1^{\mathrm{P} 288 \mathrm{~A}}$ showed significantly greater incorporation into SGs (Figure $5, \mathrm{~A}$ and $\mathrm{B}$ ). This result is consistent with previous reports that mutations in the hnRNPA1 PY-NLS reduce hnRNPA1 nuclear import and enhance incorporation into SGs $(13,14,57)$. With heat stress, we observed greater WT hnRNPA1 incorporation into SGs (Supplemental Figure 2). The *321Eext*6 mutation did not affect hnRNPA1 incorporation into heat shock-induced SGs, whereas hnRNPA1 $1^{\mathrm{P} 288 \mathrm{~A}}$ showed significantly greater incorporation (Supplemental Figure 2). G304Nfs*3 reduced incorporation of hnRNPA1 into SGs upon heat shock, suggesting that P288A and G304Nfs*3 mutations have opposite effects on hnRNPA1 incorporation into SGs.

To assess how hnRNPA1 mutations affect SG dynamics, we established a live-cell assay that monitors SG assembly and disassembly kinetics during a controlled heat pulse. Thus, we generated a U2OS cell line in which CRISPR/Cas9 was used to tag endogenous G3BP1 (a SG marker) with tdTomato (58). We transiently transfected these cells with EGFP-tagged hnRNPA1 (WT or mutant forms). Although none of the hnRNPA1 mutants had a significant effect on the number or assembly rate of SGs, we observed mutation-dependent effects on SG disassembly kinetics. When SGs were induced by a 30-minute heat shock, the hnRNPA1 $1^{\mathrm{P} 288 \mathrm{~A}}$ slightly delayed disassembly relative to WT hnRNPA1 (Figure 5C). Extending the heat shock to 60 minutes further delayed SG disassembly for hnRNPA ${ }^{\text {P288A }}$, and now hnRNPA ${ }^{\star 321 E e x t^{*} 6}$ also decelerated SG disassembly (Figure 5, D and E). Cells expressing WT hnRNPA1 or hnRNPA1 ${ }^{\mathrm{G} 304 \mathrm{Nfs}^{*} 3}$ showed nearly complete SG disassembly after 60-minute recovery (Figure 5, D and E). Consistent with our pure protein data (Figure 4D), hnRNPA1 ${ }^{\mathrm{G} 304 \mathrm{Nfs}^{*} 3}$ elicited faster SG disassembly than WT hnRNPA1 (Figure $5, \mathrm{D}$ and E). However, approximately $50 \%$ of cells expressing hnRNPA $1^{\text {P288A }}$ or hnRNPA1 $1^{* 321 \text { Eext }^{* 6}}$ failed to disassemble SGs in this time frame (Figure 5, D and E). This delay suggests that incorporation of hnRNPA1 $1^{\mathrm{P} 288 \mathrm{~A}}$ or hnRNPA1 ${ }^{* 321 \mathrm{Eext} \mathrm{t}^{*} 6}$ into SGs alters their dissolution. Moreover, these findings suggest that the severity of stress can reveal differences between hnRNPA1 variants. 
A

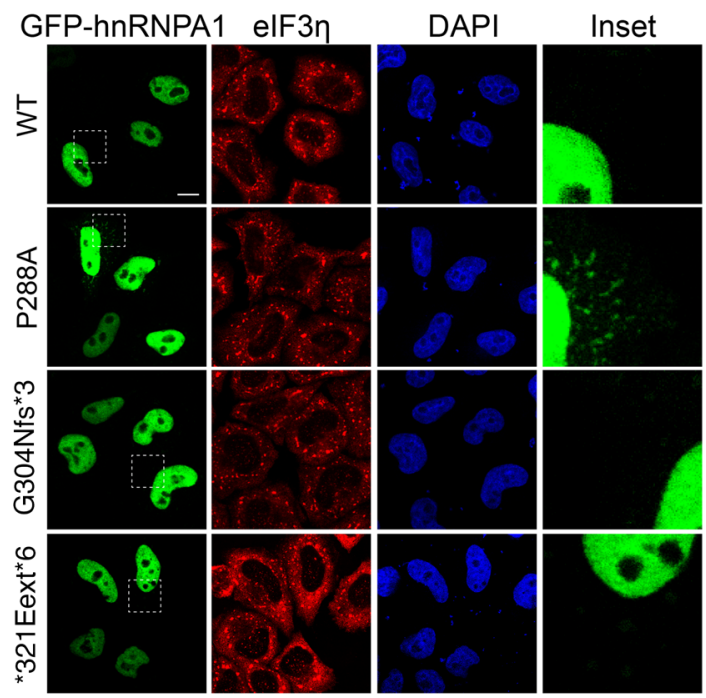

B

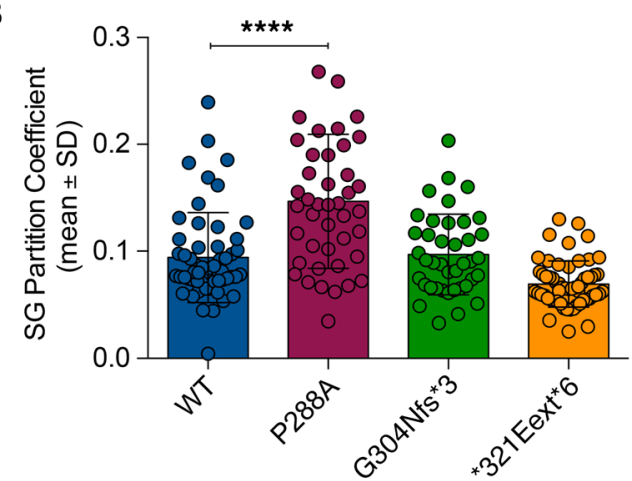

C

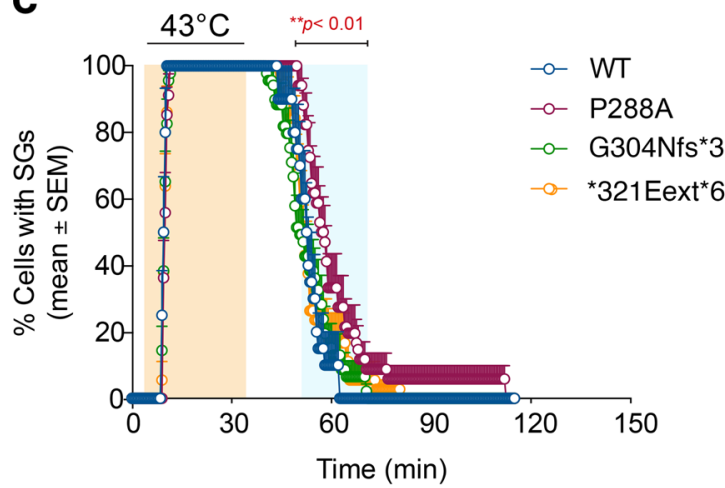

D
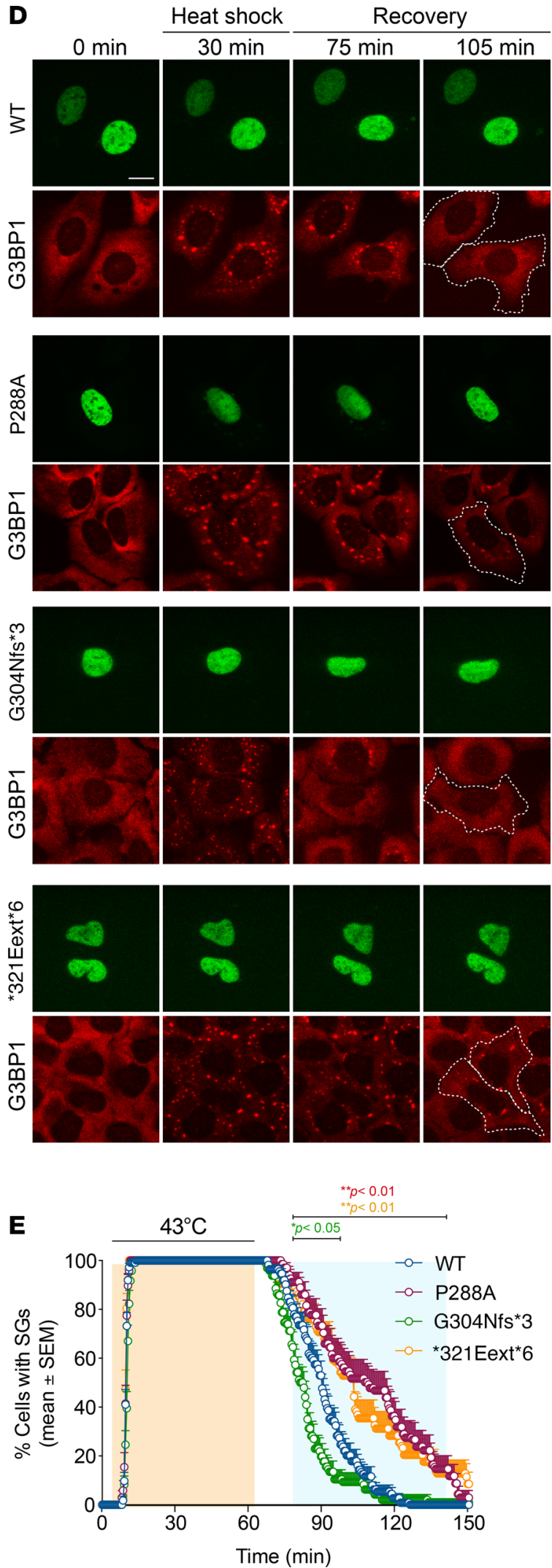

Figure 5. Localization and stress granule dynamics of the hnRNPA1 mutants. (A and B) HeLa cells were transiently transfected with WT or mutant EGFPtagged hnRNPA1 and subjected to arsenite stress ( $0.5 \mathrm{mM}$ sodium arsenite, 30 minutes). Cells were fixed and stained with elF3ๆ (red) and DAPI (blue). Confocal images were taken for partition coefficient analysis. Scale bar: $10 \mu \mathrm{m}$ (original magnification, $\times 10$ for the insets). Error bars represent mean \pm SD ( $n=55,43,40$, and 58 cells for WT, P288A, G304Nfs ${ }^{*} 3$, and ${ }^{*} 321$ Eext ${ }^{*} 6$, respectively). ${ }^{* * *} P<0.0001$, by ordinary 1 -way ANOVA with Dunnett's multiple comparisons test. (C) U2OS cells expressing tdTomato-tagged endogenous G3BP1 were transiently transfected with WT or mutant EGFP-tagged hnRNPA1 
and subjected to heat shock $\left(43^{\circ} \mathrm{C}, 30\right.$ minutes; orange shading) and allowed to recover at $37^{\circ} \mathrm{C}$ for 2 hours. Line graph represents the percentage of cells with visible tdTomato-C3BP1 puncta over time. Error bars represent mean \pm SEM $(n=10,17,23$, and 18 videos for WT, P288A, C304Nfs*3, and *321Eext*6, respectively). Blue shaded area indicates time points at which P288A mutant was statistically significantly different from WT. ${ }^{*} P<0.01$ by 2 -way ANOVA with Dunnett's multiple comparisons test. ( $\mathbf{D}$ and $\mathbf{E})$ U2OS cells expressing tdTomato-tagged endogenous C3BP1 were transiently transfected as in $\mathbf{C}$ and subjected to heat shock $\left(43^{\circ} \mathrm{C}, 60\right.$ minutes; orange shading) and allowed to recover at $37^{\circ} \mathrm{C}$ for 90 minutes. White dotted lines (D) delineate hnRNPA1-positive cells; scale bar indicates $10 \mu \mathrm{m}$. Line graph (E) represents the percentage of cells with visible tdTomato- $\mathbf{3 B P 1}$ puncta over time ( $n=56$, 34, 48, and 35 videos for WT, P288A, G304Nfs*3, and ${ }^{*} 321 \mathrm{Eext}^{*} 6$, respectively). Blue shaded area indicates time points at which each mutant was statistically significantly different from WT. ${ }^{*} P<0.05,{ }^{* *} P<0.01$ (colors correspond to the respective mutants), by 2 -way ANOVA with Dunnett's multiple comparisons test.

Finally, we evaluated how hnRNPA1 ${ }^{\text {D262V }}$, which is associated with MSP and was identified in family E in this study, altered SG formation (10). As with the other mutations tested here, hnRNPA1 incorporation into SGs was similar for WT hnRNPA1 and hnRNPA1 ${ }^{\text {D262V }}$ (Supplemental Figure 3A). However, similar to hnRN$\mathrm{PA}^{\mathrm{P} 288 \mathrm{~A}}$ or hnRNPA1 $1^{* 321 \mathrm{Eext}^{*} 6}$, hnRNPA1 $^{\text {D262V }}$ significantly delayed SG disassembly (Supplemental Figure 3 , B and C). Thus, hnRNPA1 mutations can have variable effects on SG disassembly kinetics. Specifically, P288A, D262V, and *321Eext*6 can delay SG disassembly, whereas G304Nfs*3 can accelerate SG disassembly.

\section{Discussion}

Here, we identified and characterized potentially novel and previously described HNRNPA1 mutations in patients with atypical ALS, HMN, distal myopathy, and MSP3 (Supplemental Table 2). In 6 families, we identified stop-loss mutations ${ }^{*} 321 \mathrm{Eext}^{*} 6$ and ${ }^{*} 321 \mathrm{Q}$ ext*6 leading to extension of the protein; a splice site mutation and a $500 \mathrm{bp}$ deletion, both leading to skipping of the last exon, resulting in a truncated protein, G304Nfs*3; a known missense mutation causal for ALS (P288A) in a family with an atypical slowly progressive ALS phenotype; and the known MSP3 D262V variant in a family with myopathy (14). These mutations are all in the hnRNPA1 PrLD, supporting previous findings that PrLD alterations can have important biological consequences $(10,50)$. Despite the larger cohort that was surveyed, we only identified $4 H N R N P A 1$ mutations, suggesting that $H N R N P A 1$ mutations are rare.

Mutations in HNRNPA1, though rare, show great clinical heterogeneity. Patients in family A (G304Nfs*3) and B (P288A) present with phenotypes of pure motor neuron disorders without sensory involvement and with pyramidal, bulbar, and upper motor neuron involvement spanning the spectrum of dHMN to atypical (juvenile) ALS. The slow progression of disease in these patients is striking. Patients in family B remain ambulant even 15-20 years after disease onset, unlike typical ALS cases. Patients in family C $\left({ }^{*} 321\right.$ Eext*6) present a phenotype that is compatible with a distal-onset myopathy with facial weakness with relatively faster progression, with the eldest patient using a wheelchair by age 34. Some neurogenic features suggest an overlap with the motor neuron spectrum in this family. Family F $(* 321$ Qext*6) shows a myopathic phenotype with pronounced facial weakness similar to family $\mathrm{C}$. These families harbor the similar mutations * $321 \mathrm{Eext} 6$ (family C) and *321Qext*6 (family F). The myopathic phenotype in family E (D262V) is similar to the previously reported family carrying the D262V mutation. These families demonstrate that a broader range of phenotypes is associated with pathogenic HNRNPA1 variants than was previously appreciated. Based on these findings, we suggest that it could be beneficial to test patients with a range of motor neuron diseases, including dHMN, atypical and typical ALS, as well as (distal) myopathy and MSP phenotypes, for pathogenic $H N R N$ $P A 1$ variants. Defining the mechanism by which hnRNPA1 mutations manifest pathologically is essential for understanding and treating diseases in which these mutations are present.

Several RBPs with PrLDs, including hnRNPA1, are implicated in neurodegenerative diseases $(8,10,35$, 47, 59-63). Disease-linked PrLD mutations can exacerbate the propensity of the RBP to form self-seeding fibrils, resulting in cytoplasmic aggregation and persistent SGs $(8,10,23,41)$. In addition to negative gain-offunction consequences of PrLD mutations, there are also loss-of-function consequences to having a mutated PrLD. In the case of hnRNPA1, the PrLD participates in pre-mRNA splicing, stable RNA binding, optimal RNA annealing activity, and protein-protein interactions $(64,65)$. Thus, PrLD mutations may also perturb normal hnRNPA1 function.

We assessed the intrinsic fibrillization and LLPS propensity of 4 of the HNRNPA1 mutations identified here (Table 1). P288A is predicted to accelerate fibrillization because proline can act as a gatekeeper residue that inhibits fibrillization by inducing a local twist (structural constraint) that is incompatible with cross- $\beta$ structure (49-51). ZipperDB predicted that the P288A mutation results in a new potent steric zipper in the PrLD, which we show causes more rapid fibrillization. This change in the biophysical properties of hnRNPA1 ${ }^{\mathrm{P} 288 \mathrm{~A}}$ is compounded by its impaired nuclear import (13). Indeed, the P288A mutation alters the critical proline of 
the PY-NLS of hnRNPA1, which weakens the interaction with its nuclear import receptor, karyopherin- $\beta 2$ $(\operatorname{Kap} \beta 2)(53,66)$. Thus, $\operatorname{Kap} \beta 2$ is predicted to be less able to chaperone and disaggregate hnRNPA1 ${ }^{\mathrm{P} 288 \mathrm{~A}}$ in the cytoplasm and return hnRNPA1 ${ }^{\mathrm{P} 288 \mathrm{~A}}$ to the nucleus (66). Thus, enhanced intrinsic fibrillization and reduced interaction with Kap $\beta 2$ likely combine to render P288A pathogenic. In this way, the hnRNPA1 ${ }^{\mathrm{P} 288 \mathrm{~A}}$ variant resembles ALS-linked FUS ${ }^{\mathrm{P} 525 \mathrm{~L}}$ where the critical proline of the FUS PY-NLS is mutated to leucine, which reduces the interaction with $\operatorname{Kap} \beta 2(66,67)$ and simultaneously directly promotes FUS aggregation $(68)$.

ZipperDB predicted that the $* 321$ Eext $^{*} 6$ mutation does not alter the steric zipper landscape of $\mathrm{hnRN}$ PA1. Accordingly, hnRNPA1 $1^{\star_{321 E e x}^{*} 6}$ exhibited similar fibrillization propensity as WT hnRNPA1. Finally, ZipperDB revealed that the G304Nfs*3 mutation deletes several potent steric zippers from the PrLD, which would reduce the multivalency of the PrLD and reduce fibrillization. Indeed, hnRNPA1 ${ }^{\mathrm{G} 304 \mathrm{Nfs}^{\star 3}}$ exhibited reduced fibrillization in vitro. The decelerated fibrillization of G304Nfs*3 is also observed in other mutant proteins connected with neurodegenerative disease. For example, the $\alpha$-synuclein variant, A30P, which causes familial Parkinson's disease (69), assembles into fibrils more slowly than WT $\alpha$-synuclein (70). However, $\alpha$-synuclein $^{\mathrm{A} 30 \mathrm{P}}$ accesses toxic oligomeric forms more rapidly than WT $\alpha$-synuclein and populates these forms for longer before forming fibrils (70). A similar situation could arise with G304Nfs*3, with the extended lag phase of fibrillization causing prolonged exposure to toxic oligomeric species.

In addition to forming stable fibrils, the hnRNPA1 PrLD promotes LLPS, which contributes to SG assembly $(25,54)$. hnRNPA $1^{\text {G304Nfs*3 }}$ exhibited reduced LLPS propensity, whereas hnRNPA1 ${ }^{\text {P288A }}$ and hnRN$\mathrm{PA}^{\star 321 \text { Eext*6}}$ were more similar to WT hnRNPA1. The reduced LLPS propensity of hnRNPA1 ${ }^{\mathrm{G} 304 \mathrm{Nf}{ }^{* 3}}$ could lead to loss of hnRNPA1 function for processes that require hnRNPA1 LLPS, such as (but not limited to) maintaining SG stability (71-74).

Upon stress, hnRNPA1 partitions into cytoplasmic SGs, only to disperse again once stress dissipates. HNRNPA1 mutations also affect SG dynamics. Fibrillization-prone hnRNPA1 ${ }^{\mathrm{P} 288 \mathrm{~A}}$ increased hnRNPA1 accumulation in SGs and decelerated SG disassembly. Conversely, hnRNPA1 ${ }^{\mathrm{G} 304 \mathrm{Nfs}{ }^{* 3}}$, which shows impaired LLPS and fibrillization, facilitated more rapid SG disassembly. hnRNPA ${ }^{\star 321 E e x x^{\star 6} 6}$ exhibited similar fibrillization propensity to WT hnRNPA1 but delayed SG disassembly. hnRNPA1 ${ }^{\star 321 E e x{ }^{\star} 6}$ might slow SG disassembly via the C-terminal -ELGNKA extension, which may enable SG-stabilizing interactions.

Intracellular RBP aggregates are a hallmark of ALS/FTD. However, different hypotheses have been advanced to explain how mutations in these proteins cause neurodegeneration. On the one hand, pathological aggregates can cause a toxic gain of function (75). On the other hand, mutations can lead to loss of normal function (75). Loss of hnRNPA1 function could affect splicing, translation, miRNA biogenesis, and transcription of many targets.

Our findings suggest that disease-linked HNRNPA1 mutations can have different effects on fibrillization, LLPS, and SG dynamics while still causing phenotypes within the known spectrum of disease. Thus, rather than a universal mechanism defined by accelerated fibrillization as with D262V, P288A, and D262N (10), it seems likely that there are multiple disease-causing mechanisms at work as suggested by the *321Eext* 6 and G304Nfs*3 variants. Which critical hnRNPA1 functions are most affected and how they affect physiological pathways remains to be investigated. Further research into the differences in normal functions affected by the mutations might help explain the phenotypic variation observed for HNRNPA1 mutations. The identification of additional mutations in hnRNPA1 in degenerative disorders of motor neurons and muscle will also help understand the underlying pathomechanisms. Based on our results, we suggest that there is not one unifying mechanism but rather a spectrum of disturbances, including fibrillization propensity, LLPS propensity, and SG dynamics, each of which may elicit cellular dysfunction and degeneration.

\section{Methods}

Study participants. We describe 6 families: 3 with isolated patients and 3 with a dominant inheritance of motor neuron or muscle disorders (Supplemental Table 2). Samples from these 6 families were subjected to next-generation sequencing. Additionally, 547 patients diagnosed with dHMN, CMT2, CMTi, SMA, ALS, HSP, or myopathy were screened for HNRNPA1 mutations. For all patients and family members, high-molecular-weight genomic DNA was extracted from blood samples using standard methods.

Next-generation sequencing. Next-generation sequencing (WES or WGS) was performed on high-molecular-weight genomic DNA from families A-F (Supplemental Table 1). Subsequent processing and filtering were performed in the separate research or diagnostic facilities using similar approaches and general practices used for identification of rare disease-causing genetic variants (Supplemental Table 1). 
The de novo heterozygous $500 \mathrm{bp}$ deletion identified in family $\mathrm{D}$ encompassing exon 9 of HNRNPA1 (NM_002136.4: c.907+15_*5-68del) $(76,77)$ was confirmed via a quantitative PCR assay. Transcriptome sequencing (RNA-Seq) was performed on D:II:1 cultured fibroblasts to assess the effect of the deletion on the HNRNPA1 mRNA transcript (78). RNA-Seq revealed skipping of exon 9 (https://varsome.com/transcript/ hg19/NM_002136.4), which is predicted to result in a truncated protein (p.G304Nfs*3) (Figure 2 and Supplemental Figure 1D). Additionally, RNA-Seq did not show a decrease in HNRNPA1 mRNA expression levels compared with controls, indicating the aberrant transcript escapes NMD (Supplemental Figure 1D).

Requests for deidentified sequencing data sets for the families included in this study should be directed to the corresponding author, JB.

HNRNPA1 variant screening. A Multiplex Amplification of Specific Targets for Resequencing (Agilent) assay was designed to screen for mutations in all coding regions and exon-intron boundaries of the $H N R N$ $P A 1$ gene in an additional 547 patients. Multiplex PCRs and following barcoding steps were performed on a Veriti AB machine (Life Technologies). Libraries were sequenced on a MiSeq Platform (Illumina) using v3 chemistry with read lengths of $2 \times 300 \mathrm{bp}$. For read alignment, variant calling, annotation, and filtering, GATK, SAMtools, and GenomeComb were used. For possible pathogenic HNRNPA1 variants, WES was performed to exclude other pathogenic variants, and Sanger sequencing was used for validation and segregation analysis in all available family members.

Plasmid generation. mRNA for index patient family A, index patient family $\mathrm{C}$, and a control individual was extracted from lymphoblast cell lines by RNeasy Mini Kit (QIAGEN). cDNA was generated by reverse transcription using the SuperScript III First-Strand Synthesis System (Thermo Fisher Scientific). cDNA was used for PCR amplification of HNRNPA1 (NM_002136.3) with primers containing sequences suitable for subsequent Gateway Cloning Technology (Thermo Fisher Scientific). pDONR221 was used as the entry vector. The P288A variant from family B was introduced into the WT HNRNPA1 entry clone by site-directed mutagenesis (79). The G304Nfs*3 mutation from families A and D and the *321Eext*6 variant from family $\mathrm{C}$ were cloned by direct amplification of the patient cDNA. The mRNA for the variant from family $\mathrm{D}$ is the same as the variant for family A, and thus the same plasmid was used. The entry clones were subcloned into a pLenti backbone with N-terminal V5- or EmGFP-tag for stable transduction and puromycin selection. Templates were checked by Sanger sequencing.

Yeast plasmids with each hnRNPA1 variant were generated by cloning into the pAG416GAL-GFP-ccdB vector backbone using Gateway reactions (Thermo Fisher Scientific). Mutations were generated via QuikChange Site-Directed Mutagenesis Kit (Agilent) and verified by DNA sequencing. Plasmids for bacterial protein expression were generated by cloning into a pDUET-GST vector backbone. Mutations were made via QuikChange Site-Directed Mutagenesis Kit (Agilent) and verified by DNA sequencing.

Mammalian cell culture. HeLa (CCL-2) and U2OS (HTB-96) cells were from ATCC; cultured in DMEM (HyClone) supplemented with 10\% fetal bovine serum (FBS; HyClone; SH30396.03), 1× GlutaMAX (Thermo Fisher Scientific; 35050061), and $50 \mathrm{U} / \mathrm{mL}$ penicillin and $50 \mu \mathrm{g} / \mathrm{mL}$ streptomycin (Gibco; 15140-122); and maintained at $37^{\circ} \mathrm{C}$ in a humidified incubator with $5 \% \mathrm{CO}_{2}$. U2OS cells expressing tdTomato-tagged endogenous G3BP1 have been described (58). Cells were authenticated by short tandem repeat profiling.

Western blot. HeLa cells were incubated at $37^{\circ} \mathrm{C}$ in a humidified atmosphere with $5 \% \mathrm{CO}_{2}$. Cells were cultured in MEM, with L-glutamine and Earle's salts (Thermo Fisher Scientific), with 10\% heat-inactivated FBS, 1\% L-glutamine, and 1\% penicillin-streptomycin. Transient transfection of HeLa cells with WT and mutant HNRNPA1 lentiviral plasmids was performed using polyethylenimine. Transfected HeLa cells were cultured for 24 hours before collection.

After transfection, HeLa cells $\left(4 \times 10^{6}\right)$ were pelleted and lysed with RIPA lysis buffer $(150 \mathrm{mM} \mathrm{NaCl}$, $0.5 \%$ sodium deoxycholate, $0.1 \%$ sodium dodecyl sulfate [SDS], $1 \% \mathrm{NP}-40,50 \mathrm{mM}$ Tris- $\mathrm{HCl} \mathrm{pH}$ 7.4) supplemented with protease inhibitors (Roche Diagnostics). A total of $12.5 \mu$ g protein was run on a NuPAGE Novex 4\%-12\% Bis-Tris Protein Gel (Thermo Fisher Scientific). Primary antibody mouse anti-V5 (1:5000; Life Technologies R960-25) and secondary goat anti-mouse IgG2a horseradish peroxidase (1:10,000; Southern Biotech, catalog 1081-05) were used, and visualization was performed with enhanced chemiluminescence detection (Pierce ECL Plus Western Blotting Substrate, Thermo Fisher Scientific). Equal loading was assessed by rabbit $\alpha$-tubulin primary antibody (1:5000; Abcam rabbit polyclonal IgG ab4074) with goat anti-rabbit IgG horseradish peroxidase secondary antibody (1:10,000; Southern Biotech, catalog 4030-05).

Yeast cells transformed with the appropriate plasmid were grown at $30^{\circ} \mathrm{C}$ in galactose-containing media for 8 hours to induce protein expression. Cultures were normalized to an $\mathrm{OD}_{600}=0.6$, and $6 \mathrm{~mL}$ of cells 
were harvested. Yeast lysates were extracted by incubation with $0.1 \mathrm{M} \mathrm{NaOH}$ at room temperature for 5 minutes. Lysates were mixed with SDS-sample buffer, boiled for 5 minutes, and subjected to Tris-HCl SDS-PAGE (4\%-20\% gradient, Bio-Rad), followed by transfer to a PVDF membrane (MilliporeSigma). Membranes were blocked in Odyssey blocking buffer (LI-COR) for 1 hour at room temperature. Primary antibody incubations were performed at $4^{\circ} \mathrm{C}$ overnight. After washing with PBS with $1 \%$ Tween (PBST), membranes were incubated with fluorescently labeled secondary antibodies at room temperature for 1 hour, followed by washing with PBST. Proteins were detected using an Odyssey Fc dual-mode imaging system (LI-COR). Antibodies used were rabbit polyclonal anti-GFP (MilliporeSigma, catalog G1544), mouse monoclonal anti-PGK1 (Thermo Fisher Scientific, catalog 459250), and fluorescently labeled anti-mouse and anti-rabbit secondary antibodies (LI-COR, catalog nos. 926-32210 and 926-68071, respectively).

Yeast transformation and spotting assays. All experiments were performed using Saccharomyces cerevisiae strain BY4741 (MATa, his3 $\Delta 1$, leu2 $\Delta 0$, met15 $\Delta 0$, ura $\Delta 30$ ) (80). The PEG/lithium acetate method was used to transform yeast with plasmid DNA (81). For spotting assays, yeast cells were grown overnight at $30^{\circ} \mathrm{C}$ in liquid media containing raffinose (SRaf/-Ura) until they reached log or midlog phase. Cultures were then normalized for $\mathrm{OD}_{60}$; serially diluted; spotted onto synthetic solid media containing glucose, sucrose/galactose (1:1), or galactose lacking uracil; and were grown at $30^{\circ} \mathrm{C}$ for $2-3$ days. Yeast experiments were performed in triplicate.

Protein purification. hnRNPA1 proteins were purified as described $(10,82,83)$. Protein was centrifuged at $16,100 \mathrm{~g}$ for 10 minutes at $4^{\circ} \mathrm{C}$ to remove any aggregated material before use in fibrillization and LLPS assays. After centrifugation, the protein concentration in the supernatant was determined by Bradford assay (Bio-Rad).

In vitro fibrillization assays. hnRNPA1 $(5 \mu \mathrm{M})$ fibrillization $(100 \mu \mathrm{L}$ reaction) was initiated by addition of $2 \mu \mathrm{g}$ of TEV protease in A1 assembly buffer (40 mM HEPES-NaOH at $\mathrm{pH} 7.4,150 \mathrm{mM} \mathrm{KCl,5 \%}$ glycerol, $1 \mathrm{mM}$ DTT, and $20 \mathrm{mM}$ glutathione). hnRNPA1 fibrillization reactions were incubated at $25^{\circ} \mathrm{C}$ for 24 hours with agitation at $1200 \mathrm{rpm}$ in an Eppendorf Thermomixer, at which time fibrillization was complete with approximately $100 \%$ of the hnRNPA1 in the aggregated state. For sedimentation analysis, at indicated times, fibrillization reactions were centrifuged at $16,100 \mathrm{~g}$ for 10 minutes at $4^{\circ} \mathrm{C}$. Supernatant and pellet fractions were resolved by SDS-PAGE and stained with Coomassie Brilliant Blue, and the relative amount in each fraction was determined by densitometry in Image $(\mathrm{NIH})$. Fibrillization assays were performed in triplicate. For electron microscopy, fibrillization reactions $(10 \mu \mathrm{L})$ were adsorbed onto glow-discharged 300-mesh Formvar/carbon-coated copper grids (Electron Microscopy Sciences) and stained with 2\% (w/v) aqueous uranyl acetate. Excess liquid was removed, and grids were air-dried. Samples were viewed using a JEOL 1010 transmission electron microscope.

In vitro LLPS assays. hnRNPA1 liquid droplets were formed by incubating hnRNPA1 in the absence of TEV protease at the indicated concentration in LLPS buffer (40 mM HEPES-NaOH at pH 7.4, $150 \mathrm{mM} \mathrm{NaCl}, 5 \%$ glycerol, $1 \mathrm{mM}$ DTT, and $20 \mathrm{mM}$ glutathione, $10 \%$ dextran) at $25^{\circ} \mathrm{C}$. Protein samples were spotted onto a coverslip immediately after all components had been added and imaged using a Leica DMI6000 by DIC microscopy.

Immunofluorescence. HeLa cells were seeded on 8-well glass slides (MilliporeSigma). Cells were transfected 24 hours after seeding using FuGENE 6 (Promega) with EGFP-tagged or enhanced yellow fluorescent protein-tagged (EYFP-tagged) hnRNPA1 constructs. Cells were stressed 24 hours after transfection with $500 \mu \mathrm{M}$ sodium arsenite (MilliporeSigma) for 30 minutes. Cells were then fixed with 4\% paraformaldehyde (Electron Microscopy Sciences), permeabilized with $0.5 \%$ Triton X-100, and blocked in 5\% bovine serum albumin. Primary antibodies used were against G3BP1 (611127; BD Biosciences) and eIF3ๆ (sc-16377; Santa Cruz Biotechnology). For visualization, the appropriate host-specific Alexa Fluor 555 or 647 (Molecular Probes, catalog no. A31570 or A21447, respectively) secondary antibody was used. Slides were mounted using ProLong Gold Antifade Reagent with DAPI (Life Technologies). Images were captured using a Leica TCS SP8 STED 3X confocal microscope (Leica Biosystems) with a $63 \times$ objective. Images were quantified by automated puncta analysis using CellProfiler software (Broad Institute). Cells were segmented using DAPI and G3BP1 channels, and granules were identified using G3BP1 and eIF3 $\eta$ channels. Integrated intensities of nucleus, cytoplasm, and granules were measured. Yeast cells that had been transformed with the indicated EGFP-tagged hnRNPA1 protein or vector were grown for 8 hours in galactose-containing media, pelleted, and imaged using a Leica DM IRBE microscope before being processed using ImageJ (NIH).

Live-cell time-lapse imaging. tdTomato-G3BP1-knockin U2OS cells were seeded in glass-bottomed, polyD-lysine-coated, $35 \mathrm{~mm}$ dishes (MatTek Corporation). Cells were transfected 24 hours after seeding using FuGENE 6 (Promega) with EGFP-tagged or EYFP-tagged hnRNPA1 constructs. The dish was transferred 48 hours after transfection to an Opterra II swept-field confocal microscope (Bruker) system with a stage top 
incubator with $5 \% \mathrm{CO}_{2}$ and $60 \times$ objective with an objective heater (Bioptechs), preheated to $37^{\circ} \mathrm{C}$. The system was left to equilibrate for 5-10 minutes before imaging was initiated.

For time-lapse imaging, using PrairieView software with perfect focus engaged, multipoint images were taken every 30 seconds with both 488 and $560 \mathrm{~nm}$ lasers. Two minutes into imaging, the objective temperature was raised to $43^{\circ} \mathrm{C}$ to begin heat shock; 30 or 60 minutes later, the temperature was lowered back to $37^{\circ} \mathrm{C}$ to alleviate stress, and cells were imaged until granules disappeared, or after 2-3 hours passed. Cells with equivalent expression levels of hnRNPA1 were selected for further analysis.

Live-cell analysis. Live-cell imaging analysis was performed manually. Only hnRNPA1-positive viable cells that did not have td-Tomato G3BP1-positive granules prior to the 2-minute mark were evaluated. Cells were considered granule negative at the beginning of the video and were considered granule positive at the frame in which distinct cytoplasmic granules were visible. The frame at which cytoplasmic granules were no longer visible was defined as the time point at which cells were again SG negative. Images were analyzed using Image $(\mathrm{NIH})$. The percentage of cells that were considered granule positive was determined and graphed at each time point.

Statistics. Statistical analysis was performed in GraphPad Prism. Comparisons of the mean of each group with the mean of a control group were performed by 1-way ANOVA with Dunnett's multiple comparison test. For comparing mean of control group with the means of other groups in each time point, 2-way ANOVA with either Sidak's or Dunnett's multiple-comparison test was performed. $P$ values were considered significant at values less than 0.05 unless otherwise stated.

Study approval. All patients or their legal representatives signed informed consent forms before enrollment. The ethics review boards of the University of Antwerp and Antwerp University Hospital; the Mossakowski Medical Research Centre of the Polish Academy of Sciences; the University Hospitals St-Luc of the University of Louvain; Radboud University Medical Center; the Leiden University Medical Center; Stanford University; the University of North Carolina at Chapel Hill; and Antwerp University Hospital approved this study.

\section{Author contributions}

AK, ND, PVDB, NCV, JBS, MTW, SP, PDJ, JB, and DK assessed the patients' phenotypes. D. Beijer, IM, TD, KVS, KD, RJLFL, SMVDM, D. Bonner, and AM analyzed the genetic data. HJK, LG, JS, and JPT designed experiments, and D. Beijer, HJK, LG, KO, IM, TD, CMF, LED, and AFF performed experiments. D. Beijer, HJK, JS, JPT, and JB provided critical advice and analyzed the data. D. Beijer, HJK, CMF, JS, JPT, and JB wrote the manuscript. PDJ, JS, JPT, and JB conceived the project.

\section{Acknowledgments}

We thank all the patients and family members for their participation in this study. Several authors of this publication are members of the European Reference Network for Rare Neuromuscular Diseases and of the Undiagnosed Diseases Network. This work was supported by the Association Belge contre les Maladies Neuromusculaire - Aide à la Recherche ASBL (2017-2018/05), the European Union (EU) FP7/2007-2013 under grant agreement number 2012-305121 (NEUROMICS), and the EU Horizon 2020 program (SolveRD under grant agreement 779257). JPT's funding sources include Howard Hughes Medical Institute, NIH (R35NS097974), and the St. Jude Research Collaborative on Membraneless Organelles. AK is supported by National Science Centre Poland grant 2016/23/B/NZ3/02035. SMVDM is supported by the National Institute of Neurological Disorders and Stroke/NIH P01 NS069539. JB is supported by a Senior Clinical Researcher mandate of the Research Fund - Flanders under grant agreement number $1805016 \mathrm{~N}$. JB is member of the $\mu$ NEURO Research Centre of Excellence of the University of Antwerp. JS's funding sources include Target ALS, Packard Foundation for ALS research, ALS Association, The G. Harold and Leila Y. Mathers Charitable Foundation, and NIH grant R01GM099836. LG's funding sources include an Ellison Medical Foundation/American Federation for Aging Research fellowship, an Alzheimer's Association Research fellowship, a Target ALS Springboard fellowship, and NIH grant R35GM138109. CMF's funding sources include NIH grants T32GM008275 and F31NS111870. AFF's funding sources include NIH grants T32AG00255 and F31NS087676. Research reported in this manuscript was in part supported by the NIH Common Fund, through the Office of Strategic Coordination/Office of the NIH Director under award number U01HG007708. The content is solely the responsibility of the authors and does not necessarily represent the official views of the NIH. 
Address correspondence to: Jonathan Baets, Translational Neurosciences, Faculty of Medicine, University of Antwerp — CDE, Parking 4, Building V, Universiteitsplein 1, B-2610 Wilrijk, Belgium. Phone: 32.3.265.11.02; Email: Jonathan.Baets@uantwerpen.be.

1. Park SJ, et al. Heterogeneous nuclear ribonucleoprotein A1 post-transcriptionally regulates Drp1 expression in neuroblastoma cells. Biochim Biophys Acta. 2015;1849(12):1423-1431.

2. Mohagheghi F, et al. TDP-43 functions within a network of hnRNP proteins to inhibit the production of a truncated human SORT1 receptor. Hum Mol Genet. 2016;25(3):534-545.

3. Zhao TT, et al. hnRNP A1 regulates UV-induced NF-kappaB signalling through destabilization of cIAP1 mRNA. Cell Death Differ. 2009;16(2):244-252.

4. Guil S, Caceres JF. The multifunctional RNA-binding protein hnRNP A1 is required for processing of miR-18a. Nat Struct Mol Biol. 2007;14(7):591-596.

5. Flynn RL, et al. TERRA and hnRNPA1 orchestrate an RPA-to-POT1 switch on telomeric single-stranded DNA. Nature. 2011;471(7339):532-536.

6. Lemieux B, et al. A function for the hnRNP A1/A2 proteins in transcription elongation. PLoS One. 2015;10(5):e0126654.

7. Lau JS, et al. Heterogeneous nuclear ribonucleoproteins as regulators of gene expression through interactions with the human thymidine kinase promoter. J Cell Biochem. 2000;79(3):395-406.

8. Harrison AF, Shorter J. RNA-binding proteins with prion-like domains in health and disease. Biochem J. 2017;474(8):1417-1438

9. Nedelsky NB, Taylor JP. Bridging biophysics and neurology: aberrant phase transitions in neurodegenerative disease. Nat Rev Neurol. 2019;15(5):272-286.

10. Kim HJ, et al. Mutations in prion-like domains in hnRNPA2B1 and hnRNPA1 cause multisystem proteinopathy and ALS. Nature. 2013;495(7442):467-473.

11. Benatar M, et al. Motor neuron involvement in multisystem proteinopathy: implications for ALS. Neurology. 2013;80(20):1874-1880

12. Keogh MJ, et al. Genetic compendium of 1511 human brains available through the UK Medical Research Council Brain Banks Network Resource. Genome Res. 2017;27(1):165-173.

13. Liu Q, et al. Whole-exome sequencing identifies a missense mutation in hnRNPA1 in a family with flail arm ALS. Neurology. 2016;87(17):1763-1769.

14. Naruse $\mathrm{H}$, et al. Molecular epidemiological study of familial amyotrophic lateral sclerosis in Japanese population by whole-exome sequencing and identification of novel HNRNPA1 mutation. Neurobiol Aging. 2018;61:255.e9-255.e16.

15. Calini D, et al. Analysis of hnRNPA1, A2/B1, and A3 genes in patients with amyotrophic lateral sclerosis. Neurobiol Aging. 2013;34(11):2695.e11-2695.e12.

16. Soong BW, et al. Extensive molecular genetic survey of Taiwanese patients with amyotrophic lateral sclerosis. Neurobiol Aging. 2014;35(10):2423.e1-2423.e6.

17. Le Ber I, et al. hnRNPA2B1 and hnRNPA1 mutations are rare in patients with "multisystem proteinopathy" and frontotemporal lobar degeneration phenotypes. Neurobiol Aging. 2014;35(4):934.e5-6.

18. Seelen M, et al. No mutations in hnRNPA1 and hnRNPA2B1 in Dutch patients with amyotrophic lateral sclerosis, frontotemporal dementia, and inclusion body myopathy. Neurobiol Aging. 2014;35(8):1956.e9-1956.e11.

19. Kierdaszuk B, et al. Sporadic inclusion body myositis: clinical, pathological, and genetic analysis of eight Polish patients. Folia Neuropathol. 2015;53(4):355-366.

20. Bentmann E, et al. Stress granules in neurodegeneration--lessons learnt from TAR DNA binding protein of $43 \mathrm{kDa}$ and fused in sarcoma. FEBS J. 2013;280(18):4348-4370.

21. Bosco DA, et al. Mutant FUS proteins that cause amyotrophic lateral sclerosis incorporate into stress granules. Hum Mol Genet. 2010;19(21):4160-4175.

22. Guzikowski AR, et al. Stress-induced mRNP granules: form and function of processing bodies and stress granules. Wiley Interdiscip Rev RNA. 2019;10(3):e1524.

23. Li YR, et al. Stress granules as crucibles of ALS pathogenesis. J Cell Biol. 2013;201(3):361-372.

24. Mackenzie IR, et al. TIA1 mutations in amyotrophic lateral sclerosis and frontotemporal dementia promote phase separation and alter stress granule dynamics. Neuron. 2017;95(4):808-816.

25. Molliex A, et al. Phase separation by low complexity domains promotes stress granule assembly and drives pathological fibrillization. Cell. 2015;163(1):123-133.

26. Zhang P, et al. Chronic optogenetic induction of stress granules is cytotoxic and reveals the evolution of ALS-FTD pathology. Elife. 2019;8:e39578.

27. Zhang X, et al. In vivo stress granule misprocessing evidenced in a FUS knock-in ALS mouse model. Brain. 2020;143(5):1350-1367.

28. Namkoong S, et al. Systematic characterization of stress-induced RNA granulation. Mol Cell. 2018;70(1):175-187.

29. Khong A, et al. The stress granule transcriptome reveals principles of mRNA accumulation in stress granules. Mol Cell. 2017;68(4):808-820.

30. Markmiller S, et al. Context-dependent and disease-specific diversity in protein interactions within stress granules. Cell. 2018;172(3):590-604.

31. Wheeler JR, et al. Distinct stages in stress granule assembly and disassembly. Elife. 2016;5:e18413.

32. Kedersha NL, et al. RNA-binding proteins TIA-1 and TIAR link the phosphorylation of eIF-2 alpha to the assembly of mammalian stress granules. J Cell Biol. 1999;147(7):1431-1442.

33. Ramaswami M, et al. Altered ribostasis: RNA-protein granules in degenerative disorders. Cell. 2013;154(4):727-736

34. Hackman P, et al. Welander distal myopathy is caused by a mutation in the RNA-binding protein TIA1. Ann Neurol. 2013;73(4):500-509.

35. Kwiatkowski TJ Jr, et al. Mutations in the FUS/TLS gene on chromosome 16 cause familial amyotrophic lateral sclerosis. Science. 
2009;323(5918):1205-1208.

36. Vance C, et al. Mutations in FUS, an RNA processing protein, cause familial amyotrophic lateral sclerosis type 6 . Science. 2009;323(5918):1208-1211.

37. Johnson JO, et al. Mutations in the Matrin 3 gene cause familial amyotrophic lateral sclerosis. Nat Neurosci. 2014;17(5):664-666

38. Al-Saif A, et al. A mutation in sigma-1 receptor causes juvenile amyotrophic lateral sclerosis. Ann Neurol. 2011;70(6):913-919.

39. Munch C, et al. Point mutations of the p150 subunit of dynactin (DCTN1) gene in ALS. Neurology. 2004;63(4):724-726.

40. Chen YZ, et al. DNA/RNA helicase gene mutations in a form of juvenile amyotrophic lateral sclerosis (ALS4). Am J Hum Genet. 2004;74(6):1128-1135.

41. Geuens T, et al. The hnRNP family: insights into their role in health and disease. Hum Genet. 2016;135(8):851-867.

42. Gilpin KM, et al. ALS-linked mutations in ubiquilin-2 or hnRNPA1 reduce interaction between ubiquilin- 2 and hnRNPA1. Hum Mol Genet. 2015;24(9):2565-2577.

43. Izumi R, et al. Isolated inclusion body myopathy caused by a multisystem proteinopathy-linked hnRNPA1 mutation. Neurol Genet. 2015;1(3):e23.

44. Suel KE, et al. Modular organization and combinatorial energetics of proline-tyrosine nuclear localization signals. PLoS Biol. 2008;6(6):e137.

45. Michael WM, et al. Signal sequences that target nuclear import and nuclear export of pre-mRNA-binding proteins. Cold Spring Harb Symp Quant Biol. 1995;60:663-668.

46. Truant $\mathrm{R}$, et al. Identification and functional characterization of a novel nuclear localization signal present in the yeast Nab2 poly(A)+ RNA binding protein. Mol Cell Biol. 1998;18(3):1449-1458.

47. Couthouis J, et al. A yeast functional screen predicts new candidate ALS disease genes. Proc Natl Acad Sci U S A 2011;108(52):20881-20890.

48. Goldschmidt L, et al. Identifying the amylome, proteins capable of forming amyloid-like fibrils. Proc Natl Acad Sci U S A 2010;107(8):3487-3492.

49. Richardson JS, Richardson DC. Natural beta-sheet proteins use negative design to avoid edge-to-edge aggregation. Proc Natl Acad Sci U S A. 2002;99(5):2754-2759.

50. Shorter J, Taylor JP. Disease mutations in the prion-like domains of hnRNPA1 and hnRNPA2/B1 introduce potent steric zippers that drive excess RNP granule assembly. Rare Dis. 2013;1:e25200.

51. Wang L, et al. Gatekeeper residues in the major curlin subunit modulate bacterial amyloid fiber biogenesis. Proc Natl Acad Sci U S A. 2010;107(1):163-168..

52. Siomi H, Dreyfuss G. A nuclear localization domain in the hnRNP A1 protein. J Cell Biol. 1995;129(3):551-560.

53. Lee BJ, et al. Rules for nuclear localization sequence recognition by karyopherin beta 2. Cell. 2006;126(3):543-558.

54. Lin Y, et al. Formation and maturation of phase-separated liquid droplets by RNA-binding proteins. Mol Cell. 2015;60(2):208-219.

55. Allemand E, et al. Regulation of heterogenous nuclear ribonucleoprotein A1 transport by phosphorylation in cells stressed by osmotic shock. Proc Natl Acad Sci U S A. 2005;102(10):3605-3610.

56. Cammas A, et al. Cytoplasmic relocalization of heterogeneous nuclear ribonucleoprotein A1 controls translation initiation of specific mRNAs. Mol Biol Cell. 2007;18(12):5048-5059.

57. Michael WM, et al. A nuclear export signal in hnRNP A1: a signal-mediated, temperature-dependent nuclear protein export pathway. Cell. 1995;83(3):415-422.

58. Yang P, et al. G3BP1 is a tunable switch that triggers phase separation to assemble stress granules. Cell. 2020;181(2):325-345.

59. Banfi S, et al. Identification and characterization of the gene causing type 1 spinocerebellar ataxia. Nat Genet. 1994;7(4):513-520.

60. Couthouis J, et al. Evaluating the role of the FUS/TLS-related gene EWSR1 in amyotrophic lateral sclerosis. Hum Mol Genet. 2012;21(13):2899-2911.

61. Neumann M, et al. Ubiquitinated TDP-43 in frontotemporal lobar degeneration and amyotrophic lateral sclerosis. Science. 2006;314(5796):130-133.

62. Pulst SM, et al. Moderate expansion of a normally biallelic trinucleotide repeat in spinocerebellar ataxia type 2. Nat Genet. 1996;14(3):269-276.

63. March ZM, et al. Prion-like domains as epigenetic regulators, scaffolds for subcellular organization, and drivers of neurodegenerative disease. Brain Res. 2016;1647:9-18.

64. Mayeda A, et al. Function of conserved domains of hnRNP A1 and other hnRNP A/B proteins. EMBO J. 1994;13(22):5483-5495

65. Sahu I, et al. A novel role for the proteasomal chaperone PSMD9 and hnRNPA1 in enhancing I $\mathrm{B} \alpha$ degradation and NF- $\mathrm{B}$ activation - functional relevance of predicted PDZ domain-motif interaction. FEBS J. 2014;281(11):2688-2709.

66. Guo L, et al. Nuclear-import receptors reverse aberrant phase transitions of RNA-binding proteins with prion-like domains. Cell. 2018;173(3):677-692.

67. Zhang ZC, Chook YM. Structural and energetic basis of ALS-causing mutations in the atypical proline-tyrosine nuclear localization signal of the Fused in Sarcoma protein (FUS). Proc Natl Acad Sci U S A. 2012;109(30):12017-12021.

68. Marrone L, et al. P525L promotes the aggregation of FUS by altering its biochemical and biophysical properties. https://sciencematters. io/articles/202004000008/info. Updated May 12, 2020. Accessed June 10, 2021.

69. Kruger R, et al. Ala30Pro mutation in the gene encoding alpha-synuclein in Parkinson's disease. Nat Genet. 1998;18(2):106-108.

70. Conway KA, et al. Acceleration of oligomerization, not fibrillization, is a shared property of both alpha-synuclein mutations linked to early-onset Parkinson's disease: implications for pathogenesis and therapy. Proc Natl Acad Sci U S A. 2000;97(2):571-576.

71. Patel A, et al. A liquid-to-solid phase transition of the ALS protein FUS accelerated by disease mutation. Cell. 2015;162(5):1066-1077.

72. Wegmann S, et al. Tau protein liquid-liquid phase separation can initiate tau aggregation. EMBO J. 2018;37(7):e98049.

73. Kanaan NM, et al. Liquid-liquid phase separation induces pathogenic tau conformations in vitro. Nat Commun. 2020;11(1):2809.

74. Gui X, et al. Structural basis for reversible amyloids of hnRNPA1 elucidates their role in stress granule assembly. Nat Commun. 2019;10(1):2006.

75. Ling SC, et al. Converging mechanisms in ALS and FTD: disrupted RNA and protein homeostasis. Neuron. 2013;79(3):416-438.

76. Curnin C, et al. Machine learning-based detection of insertions and deletions in the human genome [preprint]. https://doi. 
org/10.1101/628222. Posted on bioRxiv December 25, 2019.

77. Holt JM, et al. Identification of pathogenic structural variants in rare disease patients through genome sequencing [preprint]. https://doi.org/10.1101/627661. Posted on bioRxiv May 15, 2019.

78. Fresard L, et al. Identification of rare-disease genes using blood transcriptome sequencing and large control cohorts. Nat Med. 2019;25(6):911-919

79. Zheng L, et al. An efficient one-step site-directed and site-saturation mutagenesis protocol. Nucleic Acids Res. 2004;32(14):e115

80. Guthrie C, Fink GR. Guide to yeast genetics and molecular biology. Methods Enzymol. 1991;194:1-863.

81. Ito H, et al. Transformation of intact yeast cells treated with alkali cations. J Bacteriol. 1983;153(1):163-168.

82. Sun Z, et al. Molecular determinants and genetic modifiers of aggregation and toxicity for the ALS disease protein FUS/TLS PLoS Biol. 2011;9(4):e1000614.

83. Johnson BS, et al. TDP-43 is intrinsically aggregation-prone, and amyotrophic lateral sclerosis-linked mutations accelerate aggregation and increase toxicity. J Biol Chem. 2009;284(30):20329-20339. 